\title{
Nonfragile Quantized Dissipative Filter for Nonlinear Networked Systems
}

\author{
Kewang Huang $\mathbb{D}^{1},{ }^{1}$ Jianfeng Wang, ${ }^{2}$ and Feng Pan ${ }^{2}$ \\ ${ }^{1}$ School of Internet of Things Technology, Wuxi Institute of Technology, Wuxi, Jiangsu 214121, China \\ ${ }^{2}$ Key Laboratory of Advanced Process Control for Light Industry, Ministry of Education, Jiangnan University, \\ Wuxi, Jiangsu 214122, China
}

Correspondence should be addressed to Kewang Huang; huangkw@wxit.edu.cn

Received 20 October 2018; Accepted 3 January 2019; Published 3 March 2019

Academic Editor: Xianming Zhang

Copyright (c) 2019 Kewang Huang et al. This is an open access article distributed under the Creative Commons Attribution License, which permits unrestricted use, distribution, and reproduction in any medium, provided the original work is properly cited.

\begin{abstract}
To address the problem of filter parameter perturbation in nonlinear networked systems, a nonfragile quantized dissipative filter is designed by considering the coexistence of random one-step time delay, multipacket losses, and quantization error. We acquired the sufficient conditions for the existence of filter by choosing appropriate Lyapunov function as well as utilizing linear matrix inequality. Furthermore, we obtained the parameter expressions of the designed filter. The designed filter could meet the performance requirements of stability and dissipativity for the filter error system under the condition of allowed time delays, packet loss probability, and quantization density. The effectiveness of the designed filter is verified by numerical simulation.
\end{abstract}

\section{Introduction}

The word "filter" is derived from electrical engineering, where filters are used to transform electrical signals from one form to another, especially to eliminate various frequencies in a signal. This means that the main role of the filter is to keep frequency contents in the desired band and to eliminate other parts when the external input signal passes the filter. Nowadays, the filter plays an important role in networked control systems. When information is transmitted in an infinite network with limited bandwidth, such problems as overload, packet losses, and so on will occur if the data is too large. Therefore, the signal needs to be quantized in advance. Nevertheless, signal quantization will cause quantization error unavoidably. This will influence system performance and may lead to system instability [1-6].

The modern networked control systems are developing rapidly by virtue of low cost, easy installation, high reliability/flexibility, strong fault tolerance/diagnosis ability, and convenient remote manipulation/control. In network environments, network-induced delays and packet dropouts caused by limited network bandwidth and/or congested network traffic should be taken into account [7]. The occurrence of time delays can lead to poor performance or even destroy the stability of networks $[8,9]$. Due to these problems, the signals delivered to filters may be incomplete in real implementation. Consequently, the traditional techniques for designing filters may lead to unsatisfactory performance. Many scholars investigated $H_{\infty}$ filtering and passive filtering $[5,6,10] . H_{\infty}$ filtering only extracts the gain of information, whereas passive filtering only considers phase information. The theory of dissipative systems includes some basic tools, such as passive theorem, bounded real lemma, and Kalman-Yakubovich lemma and provides an effective framework for stability analysis and design of control systems. Thus, in the past several decades, dissipativity is applied to a wide range of fields, such as systems, circuits, and complex networks [11]. However, the design of filter based on dissipative theory can consider both gain and phase information. Therefore, investigating dissipative filtering is of great significance [12-19].

Ma et al. [12] investigated the problem of robust dissipative filter for discrete systems with nonlinear factors and constant time-delay, and they obtained the sufficient conditions for the existence of the filter by constructing appropriate Lyapunov function. Lin, Yang, and Wang [14] addressed 
the problem of nonlinear fuzzy filtering with multipacket losses, whereas they obtained the sufficient conditions for the existence of the filter based on linear matrix inequality (LMI) using T-S fuzzy model. Wang, Zhang, and Han [15] solved the problem of dissipative filter by considering the existence of time-varying delay during transmission for neutral systems, and an event-triggered communication scheme was introduced to design a suitable filter. Similarly, they obtained the parameter expressions of the corresponding filter by solving LMI. Tao et al. [16] discussed the problem of dissipative filter for Markov-jump systems, and slack matrix variables were introduced to eliminate the cross-coupling between the Lyapunov matrix and system matrices. Furthermore, they also obtained the sufficient conditions for the existence of the filter by solving LMI. Yang, Yang, and Liu [18] settled the problem of stability and dissipativity for nonlinear systems with filter parameter perturbation. However, they adopted a more general type of filter gain and obtained the parameter expressions of the corresponding filter by choosing suitable Lyapunov function as well as utilizing LMI. Xia et al. [19] focused on the problem of the coexistence of mixed delays and unknown transition rates for Markov-jump systems, and they obtained the sufficient conditions for the existence of the designed filter by constructing a parameter-dependent Lyapunov-Krasovskii function based on delay-partitioning approach.

Unfortunately, time delays, packet losses, and quantization error were independently considered in the abovementioned research. However, they may coexist in real network transmission. Moreover, the performance of filter may be influenced by the change of its own parameters. Therefore, time delays, packet losses, and quantization error were simultaneously considered in this paper. We designed the corresponding nonfragile quantized dissipative filter and acquired the sufficient condition for the existence of the filter based on Lyapunov stability theory and linear matrix inequality. Furthermore, we derived the parameter expression of the filter.

The rest of this paper is organized as follows. Section 2 outlines the problem formulation. Section 3 details the design of nonfragile quantized dissipative filter. Section 4 demonstrates numerical simulation results and discussions. Section 5 concludes this paper.

\section{Problem Formulation}

Consider the following nonlinear discrete system:

$$
\begin{aligned}
\boldsymbol{x}(k+1) & =\boldsymbol{A} \boldsymbol{x}(k)+\boldsymbol{B} \boldsymbol{w}(k)+\boldsymbol{f}(k, \boldsymbol{x}(k)) \\
\boldsymbol{y}(k) & =\boldsymbol{C} \boldsymbol{x}(k)+\boldsymbol{D} \boldsymbol{w}(k) \\
\boldsymbol{z}(k) & =\boldsymbol{L}_{1} \boldsymbol{x}(k)+\boldsymbol{L}_{2} \boldsymbol{w}(k)
\end{aligned}
$$

where $\boldsymbol{x}(k) \in R^{n}$ is the state vector, $\boldsymbol{z}(k) \in R^{q}$ is the signal to be estimated, $\boldsymbol{y}(k) \in R^{P}$ is measurement output, $\boldsymbol{w}(k) \in$ $R^{m}$ is the disturbance, and $\boldsymbol{w}(k) \in L_{2}[0, \infty) ; \boldsymbol{A} \in R^{n \times n}, \boldsymbol{B} \in$ $R^{n \times m}, \boldsymbol{C} \in R^{n \times n}, \boldsymbol{D} \in R^{n \times m}, \boldsymbol{L}_{1} \in R^{q \times n}$, and $\boldsymbol{L}_{2} \in R^{q \times m}$ are constant matrices, respectively; $\boldsymbol{f}(k, \boldsymbol{x}(k)) \in R^{n}$ is a nonlinear vector that meets Lipschitz condition; that is, $\|\boldsymbol{f}(k, \boldsymbol{x}(k))\| \leq$ $\left\|\boldsymbol{W}_{2} \boldsymbol{x}(k)\right\| ; \boldsymbol{W}_{2}$ is a constant matrix.

Considering the limited bandwidth, the logarithmic quantizer is utilized to relieve the tension on the network channel $[1,2]$. We adopt the definition of logarithmic quantizer given in [2], and the quantization level is denoted as

$$
\begin{array}{r}
U=\left\{ \pm u_{i}, u_{i}=\rho^{i} u_{0}, i= \pm 1, \pm 2, \ldots\right\} \cup\left\{ \pm u_{(0)}\right\} \cup\{0\} \\
0<\rho<1, u_{(0)}>0 .
\end{array}
$$

The corresponding logarithmic quantizer $q(v)$ is defined as

$$
q(v)= \begin{cases}u_{i} & \text { if } \frac{1}{1+\delta} u_{i}<v<\frac{1}{1-\delta} u_{i}, v>0 \\ 0 & \text { if } v=0 \\ -q(-v) & \text { if } v<0\end{cases}
$$

where $\rho$ is quantization density, $\delta=(1-\rho) /(1+\rho)$ represents the upper bound of quantization error, and $v$ is the signal sampled before quantization.

The measurement output after quantization can be described as

$$
\widetilde{y}=q(\boldsymbol{y})
$$

Utilizing the sector boundary method, the quantization error of measurement output can be defined as

$$
e_{k} \triangleq q(y(k))-y(k) \triangleq \Delta_{k} y(k), \quad \Delta_{k} \in[-\delta, \delta]
$$

Then, (4) can be expressed as

$$
\widetilde{\boldsymbol{y}}=\left(1+\Delta_{k}\right) \boldsymbol{y}
$$

Considering that there may be a random one-step delay or multipacket losses in real received measurement output, the corresponding mathematical model can be expressed as

$$
\begin{aligned}
\bar{y}(k) & \\
= & \alpha(k) \tilde{y}(k) \\
& +(1-\alpha(k))(1-\alpha(k-1)) \beta(k) \tilde{y}(k-1) \\
& +(1-\alpha(k))[1-(1-\alpha(k-1)) \beta(k)] \bar{y}(k-1)
\end{aligned}
$$

where $\bar{y}(k) \in R^{P}$ is the real received measurement output.

Substituting (6) into (7), we have

$$
\begin{aligned}
\bar{y}(k)= & \alpha(k)\left(1+\Delta_{k}\right) \boldsymbol{y}(k)+(1-\alpha(k)) \\
& \cdot(1-\alpha(k-1)) \beta(k)\left(1+\Delta_{k}\right) \boldsymbol{y}(k-1) \\
+ & (1-\alpha(k))[1-(1-\alpha(k-1)) \beta(k)] \\
& \cdot \bar{y}(k-1)
\end{aligned}
$$

where $\alpha(k)$ and $\beta(k)$ are uncorrelated random sequences with Bernoulli distribution. 

ities:

Respectively, they meet the following statistical probabil-

$$
\begin{aligned}
& \operatorname{prob}\{\alpha(k)=1\}=\bar{\alpha}, \\
& \operatorname{prob}\{\alpha(k)=0\}=1-\bar{\alpha} \\
& \operatorname{prob}\{\beta(k)=1\}=\bar{\beta}, \\
& \operatorname{prob}\{\beta(k)=0\}=1-\bar{\beta}
\end{aligned}
$$

where $\operatorname{prob}\{\cdot\}$ represents event probability.

From (8), we have the following:

$P\{\alpha(k)=0, \alpha(k-1)=0, \beta(k)=1\}=(1-\bar{\alpha})^{2} \bar{\beta}$ is the probability of one-step time delay; $P\{\alpha(k)=0, \alpha(k-1)=$ $0, \beta(k)=0\}+P\{\alpha(k)=0, \alpha(k-1)=1\}=(1-\bar{\alpha})^{2}(1-\bar{\beta})+$ $(1-\bar{\alpha}) \bar{\alpha}$ is the probability of the packet loss; $P\{\alpha(k)=1\}=\bar{\alpha}$ is the probability of timely received data.

For convenience later, we define the following variable:

$$
\begin{aligned}
\widehat{y}(k)= & (1-\alpha(k)) \beta(k+1)\left(1+\Delta_{k}\right) \boldsymbol{y}(k) \\
& +[1-(1-\alpha(k)) \beta(k+1)] \bar{y}(k)
\end{aligned}
$$

Combining (8) and (10), we have

$$
\overline{\boldsymbol{y}}(k)=\alpha(k)\left(1+\Delta_{k}\right) \boldsymbol{y}(k)+(1-\alpha(k)) \hat{\boldsymbol{y}}(k-1)
$$

Further, combining (1), (10), and (11), we have the following augmented system:

$$
\begin{gathered}
\overline{\boldsymbol{x}}(k+1)=\overline{\boldsymbol{A}} \overline{\boldsymbol{x}}(k)+\overline{\boldsymbol{B}} \boldsymbol{w}(k)+\boldsymbol{G} \boldsymbol{f}(k, \boldsymbol{x}(k)) \\
\overline{\boldsymbol{y}}(k)=\overline{\boldsymbol{C}} \overline{\boldsymbol{x}}(k)+\overline{\boldsymbol{D}} \boldsymbol{w}(k) \\
\boldsymbol{z}(k)=\overline{\boldsymbol{L}} \overline{\boldsymbol{x}}(k)+\boldsymbol{L}_{2} \boldsymbol{w}(k)
\end{gathered}
$$

where

$$
\begin{aligned}
& \overline{\boldsymbol{x}}(k+1)=\left[\begin{array}{lll}
\boldsymbol{x}(k+1)^{T} & \hat{y}(k)^{T} & \bar{y}(k)^{T}
\end{array}\right]^{T}, \\
& \overline{\boldsymbol{x}}(k) \in R^{n+2 p}, \\
& \overline{\boldsymbol{A}}=\boldsymbol{A}_{0}+\alpha(k) \boldsymbol{A}_{1}+(1-\alpha(k)) \beta(k+1) \boldsymbol{A}_{2}, \\
& \overline{\boldsymbol{B}}=\boldsymbol{B}_{0}+\alpha(k) \boldsymbol{B}_{1}+(1-\alpha(k)) \beta(k+1) \boldsymbol{B}_{2}, \\
& \overline{\boldsymbol{C}}=\boldsymbol{C}_{0}+\alpha(k) \boldsymbol{C}_{1}, \\
& \overline{\boldsymbol{D}}=\alpha(k) \boldsymbol{D}_{1}, \\
& \overline{\boldsymbol{L}}=\left[\begin{array}{lll}
\boldsymbol{L}_{1} & \mathbf{0} & \mathbf{0}
\end{array}\right],
\end{aligned}
$$

$A_{0}=\left[\begin{array}{lll}A & 0 & 0 \\ 0 & I & 0 \\ 0 & I & 0\end{array}\right]$

$\boldsymbol{B}_{0}=\left[\begin{array}{l}\boldsymbol{B} \\ \mathbf{0} \\ \mathbf{0}\end{array}\right]$,

$C_{0}=\left[\begin{array}{lll}\mathbf{0} & \boldsymbol{I} & \mathbf{0}\end{array}\right]$,

$\boldsymbol{A}_{1}=\left[\begin{array}{ccc}0 & 0 & 0 \\ \left(1+\Delta_{k}\right) C & -I & 0 \\ \left(1+\Delta_{k}\right) C & -I & 0\end{array}\right]$,

$\boldsymbol{B}_{1}=\left[\begin{array}{c}\mathbf{0} \\ \left(1+\Delta_{k}\right) \boldsymbol{D} \\ \left(1+\Delta_{k}\right) \boldsymbol{D}\end{array}\right]$

$\boldsymbol{C}_{1}=\left[\left(1+\Delta_{k}\right) \boldsymbol{C}-\boldsymbol{I} \quad \mathbf{0}\right]$,

$\boldsymbol{A}_{2}=\left[\begin{array}{ccc}\mathbf{0} & \mathbf{0} & \mathbf{0} \\ \left(1+\Delta_{k}\right) \boldsymbol{C} & -\boldsymbol{I} & \mathbf{0} \\ \mathbf{0} & \mathbf{0} & \mathbf{0}\end{array}\right]$,

$\boldsymbol{B}_{2}=\left[\begin{array}{c}\mathbf{0} \\ \left(1+\Delta_{k}\right) \boldsymbol{D} \\ \mathbf{0}\end{array}\right]$,

$\boldsymbol{G}=\left[\begin{array}{l}\boldsymbol{I} \\ \mathbf{0}\end{array}\right]$

$\boldsymbol{D}_{1}=\left(1+\Delta_{k}\right) \boldsymbol{D}$.

\section{Nonfragile Dissipative Filter Design}

Consider designing the following filter:

$$
\begin{aligned}
\widehat{\boldsymbol{x}}(k+1) & =\boldsymbol{A}_{f d} \widehat{\boldsymbol{x}}(k)+\boldsymbol{B}_{f d} \overline{\boldsymbol{y}}(k) \\
\boldsymbol{z}_{f}(k) & =\boldsymbol{C}_{f d} \widehat{\boldsymbol{x}}(k)+\boldsymbol{D}_{f d} \overline{\boldsymbol{y}}(k)
\end{aligned}
$$

where $\hat{\boldsymbol{x}}(k) \in R^{n+p}$ is the state estimation of the filter; $\boldsymbol{z}_{f}(k) \in R^{q}$ is the estimation of $z(k)$, and $\boldsymbol{A}_{f} \in R^{(n+2 p) \times(n+2 p)}$, $\boldsymbol{B}_{f} \in R^{(n+2 p) \times p}, \boldsymbol{C}_{f} \in R^{q \times(n+2 p)}$, and $\boldsymbol{D}_{f} \in R^{q \times p}$ are parameter matrices of the filter, respectively. $\boldsymbol{A}_{f d}, \boldsymbol{B}_{f d}, \boldsymbol{C}_{f d}$, and $\boldsymbol{D}_{f d}$ can be updated as follows:

$$
\begin{aligned}
& \boldsymbol{A}_{f d}=\boldsymbol{A}_{f}+\Delta \boldsymbol{A}_{f}, \\
& \boldsymbol{B}_{f d}=\boldsymbol{B}_{f}+\Delta \boldsymbol{B}_{f} \\
& \boldsymbol{C}_{f d}=\boldsymbol{C}_{f}+\Delta \boldsymbol{C}_{f}, \\
& \boldsymbol{D}_{f d}=\boldsymbol{D}_{f}+\Delta \boldsymbol{D}_{f}
\end{aligned}
$$


where $\boldsymbol{A}_{f}, \boldsymbol{B}_{f}, \boldsymbol{C}_{f}$, and $\boldsymbol{D}_{f}$ are given parameter matrices and their corresponding perturbation can be calculated as follows:

$$
\begin{aligned}
\Delta \boldsymbol{A}_{f} & =\boldsymbol{H}_{1} \boldsymbol{F}_{1}(k) \boldsymbol{E}_{1}, \\
\Delta \boldsymbol{B}_{f} & =\boldsymbol{H}_{2} \boldsymbol{F}_{2}(k) \boldsymbol{E}_{2} \\
\Delta \boldsymbol{C}_{f} & =\boldsymbol{H}_{3} \boldsymbol{F}_{3}(k) \boldsymbol{E}_{3}, \\
\Delta \boldsymbol{D}_{f} & =\boldsymbol{H}_{4} \boldsymbol{F}_{4}(k) \boldsymbol{E}_{4}
\end{aligned}
$$

where $\boldsymbol{H}_{1} \in R^{(n+2 p) \times r}, \boldsymbol{H}_{2} \in R^{(n+2 p) \times r}, \boldsymbol{H}_{3} \in R^{n \times r}, \boldsymbol{H}_{4} \in R^{q \times r}$, $\boldsymbol{E}_{1} \in R^{r \times(n+2 p)}, \boldsymbol{E}_{2} \in R^{r \times(n+2 p)}, \boldsymbol{E}_{3} \in R^{r \times(n+2 p)}, \boldsymbol{E}_{4} \in R^{r \times p}$, and $\boldsymbol{F}_{i}(k)$ meet:

$$
\boldsymbol{F}_{i}(k)^{T} \boldsymbol{F}_{i}(k) \leq \boldsymbol{I}, \quad i=1,2,3,4
$$

Combining (12) and (14), we can obtain the following filter error system:

$$
\begin{aligned}
\widetilde{\boldsymbol{x}}(k+1) & =\widetilde{\boldsymbol{A}} \widetilde{\boldsymbol{x}}(k)+\widetilde{\boldsymbol{B}} \boldsymbol{w}(k)+\widetilde{\boldsymbol{G}} f(k, \boldsymbol{x}(k)) \\
\boldsymbol{e}(k) & =\widetilde{\boldsymbol{C}} \widetilde{\boldsymbol{x}}(k)+\widetilde{\boldsymbol{D}} \boldsymbol{w}(k)
\end{aligned}
$$

where $\tilde{\boldsymbol{x}}(k)=\left[\begin{array}{ll}\overline{\boldsymbol{x}}(k)^{T} & \widehat{\boldsymbol{x}}(k)^{T}\end{array}\right]^{T}$ and $\boldsymbol{e}(k)=\boldsymbol{z}(k)-\boldsymbol{z}_{f}(k)$ is the estimated error:

$$
\begin{aligned}
\widetilde{\boldsymbol{A}} & =\left[\begin{array}{cc}
\overline{\boldsymbol{A}} & \mathbf{0} \\
\boldsymbol{B}_{f d} \overline{\boldsymbol{C}} & \boldsymbol{A}_{f d}
\end{array}\right] \\
& =\widetilde{\boldsymbol{A}} 0+\alpha(k) \widetilde{\boldsymbol{A}}_{1}+(1-\alpha(k)) \beta(k+1) \widetilde{\boldsymbol{A}}_{2}, \\
\widetilde{\boldsymbol{D}} & =\boldsymbol{L}_{2}-\boldsymbol{D}_{f d} \boldsymbol{D} \alpha(k) \\
\widetilde{\boldsymbol{B}} & =\left[\begin{array}{cc}
\overline{\boldsymbol{B}} \\
\boldsymbol{B}_{f d} \overline{\boldsymbol{D}}
\end{array}\right] \\
& =\widetilde{\boldsymbol{B}}_{0}+\alpha(k) \widetilde{\boldsymbol{B}}_{1}+(1-\alpha(k)) \beta(k+1) \widetilde{\boldsymbol{B}}_{2} \\
\widetilde{\boldsymbol{C}} & =\left[\begin{array}{cc}
\overline{\boldsymbol{L}}_{-}-\boldsymbol{D}_{f d} & \overline{\boldsymbol{C}}-\boldsymbol{C}_{f d}
\end{array}\right]=\widetilde{\boldsymbol{C}}_{0}+\alpha(k) \widetilde{\boldsymbol{C}}_{1} \\
\widetilde{\boldsymbol{A}}_{0} & =\left[\begin{array}{cc}
\boldsymbol{A}_{0} & \mathbf{0} \\
\boldsymbol{B}_{f d} \boldsymbol{C}_{0} & \boldsymbol{A}_{f d}
\end{array}\right], \\
\widetilde{\boldsymbol{A}}_{1} & =\left[\begin{array}{cc}
\boldsymbol{A}_{1} & \mathbf{0} \\
\boldsymbol{B}_{f d} \boldsymbol{C}_{1} & \mathbf{0}
\end{array}\right],
\end{aligned}
$$

$$
\begin{aligned}
\widetilde{\boldsymbol{A}}_{2} & =\left[\begin{array}{cc}
\boldsymbol{A}_{2} & \mathbf{0} \\
\mathbf{0} & \mathbf{0}
\end{array}\right], \\
\widetilde{\boldsymbol{B}}_{0} & =\left[\begin{array}{c}
\boldsymbol{B}_{0} \\
\mathbf{0}
\end{array}\right], \\
\widetilde{\boldsymbol{B}}_{1} & =\left[\begin{array}{c}
\boldsymbol{B}_{1} \\
\boldsymbol{B}_{f d} \boldsymbol{D}
\end{array}\right] \\
\widetilde{\boldsymbol{B}}_{2} & =\left[\begin{array}{c}
\boldsymbol{B}_{2} \\
\mathbf{0}
\end{array}\right], \\
\widetilde{\boldsymbol{C}}_{0} & =\left[\begin{array}{ll}
\overline{\boldsymbol{L}}_{-}-\boldsymbol{D}_{f d} \boldsymbol{C}_{0} & -\boldsymbol{C}_{f d}
\end{array}\right], \\
\widetilde{\boldsymbol{C}}_{1} & =\left[\begin{array}{ll}
-\boldsymbol{D}_{f d} \boldsymbol{C}_{1} & \mathbf{0}
\end{array}\right], \\
\widetilde{\boldsymbol{D}} & =\boldsymbol{L}_{2}-\boldsymbol{D}_{f d} \boldsymbol{D}_{1} a(k)
\end{aligned}
$$

Defining $\stackrel{*}{\boldsymbol{A}}=E\{\widetilde{\boldsymbol{A}}\}, \stackrel{*}{\boldsymbol{B}}=E\{\widetilde{\boldsymbol{B}}\}, \stackrel{*}{\boldsymbol{C}}=E\{\widetilde{\boldsymbol{C}}\}, \stackrel{*}{\boldsymbol{D}}=$ $E\{\widetilde{\boldsymbol{D}}\}, \boldsymbol{A}_{d}=E\{\overline{\boldsymbol{A}}\}, \boldsymbol{B}_{d}=E\{\overline{\boldsymbol{B}}\}, \boldsymbol{C}_{d}=E\{\overline{\boldsymbol{C}}\}$, and $\boldsymbol{D}_{d}=$ $E\{\overline{\boldsymbol{D}}\}$ where $E$ represents expectation and letting $\langle\boldsymbol{x}, \boldsymbol{H}, \boldsymbol{y}\rangle_{t}=$ $E\left[\sum_{0}^{t} \boldsymbol{x}(k)^{T} \boldsymbol{H} \boldsymbol{y}(k)\right]$, we select the following secondary energy supply function for (18):

$$
\bar{E}(\boldsymbol{w}, \boldsymbol{e}, \boldsymbol{t})=\langle\boldsymbol{e}, \boldsymbol{Q}, \boldsymbol{e}\rangle_{t}+2\langle\boldsymbol{e}, \boldsymbol{S}, \boldsymbol{w}\rangle_{t}+\langle\boldsymbol{w}, \boldsymbol{R}, \boldsymbol{w}\rangle_{t}
$$

where $\mathbf{Q}=\boldsymbol{Q}^{T}, \boldsymbol{S}$, and $\boldsymbol{R}=\boldsymbol{R}^{T}$ are matrices of appropriate dimensions, respectively.

The aim of this paper is to design a filter by considering the coexistence of random one-step time delay, multipacket losses, and parameter perturbation. The designed filter should be able to estimate the system state and the signal to be estimated accurately, and it should meet the following two performance requirements:

(1) When $\boldsymbol{w}(k)=0$, if there exists a scalar $\chi>0$ such that the inequality $E\left[\sum_{k=0}^{\infty}\|\widetilde{\boldsymbol{x}}(k)\|^{2}\right]<\chi \sup _{-\tau<i \leq 0} E\left[\|\phi(i)\|^{2}\right]$ holds, then system (18) is stochastically stable.

(2) For any $t>0$ and nonzero $\boldsymbol{w}(k) \in L_{2}[0, \infty)$, if there exists a scalar $a>0$ such that the inequality $E\left[a \sum_{0}^{t} \boldsymbol{w}(k)^{T} \boldsymbol{w}(k)\right]<\bar{E}(w, e, t)$ holds under zero initial conditions, then system (18) is strictly $(\mathbf{Q}, S, \boldsymbol{R})$ dissipative.

\subsection{Dissipativity Analysis}

Lemma 1 (see [20]). Given matrices $\boldsymbol{N}=\boldsymbol{N}^{T}, \boldsymbol{H}$ and $\boldsymbol{E}$ are of appropriate dimensions, and matrix $\boldsymbol{F}$ meets $\boldsymbol{F}^{\boldsymbol{T}} \boldsymbol{F} \leq \boldsymbol{I}$ such that $\boldsymbol{N}+\boldsymbol{H} \boldsymbol{F} \boldsymbol{E}+\boldsymbol{E}^{T} \boldsymbol{F}^{T} \boldsymbol{H}^{T}<0$. If there exists a positive $\bar{\varepsilon}$, then the sufficient and necessary condition of $\boldsymbol{N}+\boldsymbol{H} \boldsymbol{F} \boldsymbol{E}+\boldsymbol{E}^{T} \boldsymbol{F}^{T} \boldsymbol{H}^{T}<$ 0 changes into $\boldsymbol{N}+\bar{\varepsilon} \boldsymbol{H} \boldsymbol{H}^{T}+\overline{\boldsymbol{\varepsilon}}^{-1} \boldsymbol{E}^{T} \boldsymbol{E}<0$.

The detailed proof of Lemma 1 can be found in [20]; it is omitted here.

Theorem 2. For given matrices $\mathbf{Q}=\mathbf{Q}^{T}<0, \boldsymbol{R}=\boldsymbol{R}^{T}$, and $\boldsymbol{S}$, assuming that $\boldsymbol{A}_{f d}, \boldsymbol{B}_{f d} \boldsymbol{C}_{f d}, \boldsymbol{D}_{f d}$ are known, if there exists symmetric positive definite matrix $\boldsymbol{P}$ and constant $\tau>0$ 
such that (21) holds, then system (18) is stochastically stable and strictly $(\mathbf{Q}, \mathbf{S}, \mathbf{R})$ dissipative.

$$
\left[\begin{array}{ccccccccc}
-\boldsymbol{P}+\boldsymbol{H} & \mathbf{0} & * & * & * & * & * & * & * \\
\mathbf{0} & -\tau \boldsymbol{I} & * & * & * & * & * & * & * \\
-\boldsymbol{S}^{T} \boldsymbol{C}^{*} & \mathbf{0} & -\boldsymbol{D}^{* T} \boldsymbol{S}-\boldsymbol{S}^{T} \boldsymbol{D}^{*}-\boldsymbol{R} & * & * & * & * & * & * \\
\boldsymbol{A}^{*} & \widetilde{\boldsymbol{G}} & \boldsymbol{B}^{*} & -\boldsymbol{P}^{-1} & * & * & * & * & * \\
\sigma_{1} \widetilde{\boldsymbol{A}}_{1} & \mathbf{0} & \sigma_{1} \widetilde{\boldsymbol{B}}_{1} & \mathbf{0} & -\boldsymbol{P}^{-1} & * & * & * & * \\
\sigma_{2} \widetilde{\boldsymbol{A}}_{2} & \mathbf{0} & \sigma_{2} \widetilde{\boldsymbol{B}}_{2} & \mathbf{0} & \mathbf{0} & -\boldsymbol{P}^{-1} & * & * & * \\
\sigma_{3} \widetilde{\boldsymbol{A}}_{1,2} & \mathbf{0} & \sigma_{3} \widetilde{\boldsymbol{B}}_{1,2} & \mathbf{0} & \mathbf{0} & \mathbf{0} & -\boldsymbol{P}^{-1} & * & * \\
\widetilde{\boldsymbol{C}}^{*} & \mathbf{0} & \widetilde{\boldsymbol{D}}^{*} & \mathbf{0} & \mathbf{0} & \mathbf{0} & \mathbf{0} & \mathbf{Q}^{-1} & * \\
\sigma_{4} \widetilde{\boldsymbol{C}}_{1} & \mathbf{0} & -\sigma_{4} \boldsymbol{D}_{f} \boldsymbol{D}_{1} & \mathbf{0} & \mathbf{0} & \mathbf{0} & \mathbf{0} & \mathbf{0} & \mathbf{Q}^{-1}
\end{array}\right]<0
$$

where $\widetilde{\boldsymbol{A}}_{1,2}=\widetilde{\boldsymbol{A}}_{1}-\widetilde{\boldsymbol{A}}_{2}, \sigma_{1}=\sqrt{\bar{\alpha}(1-\bar{\alpha})(1-\bar{\beta})}, \sigma_{2}=$ $\sqrt{(1-\bar{\alpha})^{2}(1-\bar{\beta}) \bar{\beta}}, \sigma_{3}=\sqrt{\bar{\alpha} \bar{\beta}(1-\bar{\alpha})}$, and $\sigma_{4}=\sqrt{\bar{\alpha}(1-\bar{\alpha})}$.

Proof. We partition the proof into two parts.

Firstly, when $\boldsymbol{w}(k)=0$, we prove that system (18) is stochastically stable.

Consider constructing the following Lyapunov function:

$$
V(\widetilde{\boldsymbol{x}}(k))=\tilde{\boldsymbol{x}}(k)^{T} P \widetilde{\boldsymbol{x}}(k)
$$

Define

$$
\begin{aligned}
E & \{\Delta V(\widetilde{\boldsymbol{x}}(k))\}+\tau \boldsymbol{x}(k)^{T} \boldsymbol{W}_{2}{ }^{T} \boldsymbol{W}_{2} \boldsymbol{x}(k) \\
& -\tau \boldsymbol{f}(k)^{T} \boldsymbol{f}(k)=E\{V(\widetilde{\boldsymbol{x}}(k+1)) \mid \widetilde{\boldsymbol{x}}(k)\} \\
& -V(\widetilde{\boldsymbol{x}}(k))+\tau \boldsymbol{x}(k)^{T} \boldsymbol{W}_{2}{ }^{T} \boldsymbol{W}_{2} \boldsymbol{x}(k) \\
& -\tau \boldsymbol{f}(k)^{T} \boldsymbol{f}(k)=\zeta(k)^{T} \boldsymbol{\Phi} \zeta(k)
\end{aligned}
$$

where

$$
\begin{aligned}
& \zeta(k)=\left[\begin{array}{ll}
\widetilde{\boldsymbol{x}}(k)^{T} & \boldsymbol{f}(k)^{T}
\end{array}\right]^{T}, \\
& \boldsymbol{\Phi}=\left[\begin{array}{cc}
-\boldsymbol{P}+\boldsymbol{H} & \mathbf{0} \\
\mathbf{0} & -\tau \boldsymbol{I}
\end{array}\right]-\left[\begin{array}{c}
\boldsymbol{\Gamma}_{1}^{T} \\
\boldsymbol{\Gamma}_{2}^{T}
\end{array}\right] \widetilde{\boldsymbol{P}}^{-1}\left[\begin{array}{ll}
\boldsymbol{\Gamma}_{1} & \boldsymbol{\Gamma}_{2}
\end{array}\right] \\
& \Gamma_{1}=\left[\begin{array}{lllll}
A^{* T} & \beta_{1} \widetilde{A}_{1}^{T} & \beta_{2} \widetilde{\boldsymbol{A}}_{2}^{T} & \sigma_{3} \widetilde{\boldsymbol{A}}_{1,2}^{T}
\end{array}\right]^{T}, \\
& \boldsymbol{\Gamma}_{2}=\left[\begin{array}{llll}
\widetilde{\boldsymbol{G}}^{T} & \mathbf{0} & \mathbf{0} & \mathbf{0}
\end{array}\right]^{T}, \\
& \widetilde{G}=\left[\begin{array}{l}
G \\
0
\end{array}\right] \\
& \boldsymbol{H}=\left[\begin{array}{cc}
\tau \boldsymbol{W}_{1}^{T} \boldsymbol{W}_{1} & \mathbf{0} \\
\mathbf{0} & \mathbf{0}
\end{array}\right], \\
& W_{1}=\left[\begin{array}{ll}
W_{2} & 0
\end{array}\right] \text {, }
\end{aligned}
$$

$$
\begin{aligned}
\widetilde{\boldsymbol{P}} & =\left[\begin{array}{cccc}
\boldsymbol{P}^{-1} & \mathbf{0} & \mathbf{0} & \mathbf{0} \\
\mathbf{0} & \boldsymbol{P}^{-1} & \mathbf{0} & \mathbf{0} \\
\mathbf{0} & \mathbf{0} & \boldsymbol{P}^{-1} & \mathbf{0} \\
\mathbf{0} & \mathbf{0} & \mathbf{0} & \boldsymbol{P}^{-1}
\end{array}\right], \\
\widetilde{\boldsymbol{A}}_{1,2} & =\widetilde{\boldsymbol{A}}_{1}-\widetilde{\boldsymbol{A}}_{2}
\end{aligned}
$$

According to Schur complement lemma, $\Phi<0$ is equivalent to the following equation:

$$
\left[\begin{array}{cccccc}
-\boldsymbol{P}+\boldsymbol{H} & * & * & * & * & * \\
\mathbf{0} & -\tau \boldsymbol{I} & * & * & * & * \\
\boldsymbol{A}^{*} & \widetilde{\mathbf{G}} & -\boldsymbol{P}^{-1} & * & * & * \\
\sigma_{1} \widetilde{\boldsymbol{A}}_{1} & \mathbf{0} & \mathbf{0} & -\boldsymbol{P}^{-1} & * & * \\
\sigma_{2} \widetilde{\boldsymbol{A}}_{2} & \mathbf{0} & \mathbf{0} & \mathbf{0} & -\boldsymbol{P}^{-1} & * \\
\sigma_{3} \widetilde{\boldsymbol{A}}_{1,2} & \mathbf{0} & \mathbf{0} & \mathbf{0} & \mathbf{0} & -\boldsymbol{P}^{-1}
\end{array}\right]<0
$$

Considering that $\boldsymbol{f}(k)^{T} \boldsymbol{f}(k) \leq \boldsymbol{x}(k)^{T} \boldsymbol{W}_{2}{ }^{T} \boldsymbol{W}_{2} \boldsymbol{x}(k)$, if there exists $\tau>0$, then the inequalities $\tau \boldsymbol{x}(k)^{T} \boldsymbol{W}_{2}{ }^{T} \boldsymbol{W}_{2} \boldsymbol{x}(k)-$ $\tau \boldsymbol{f}(k)^{T} \boldsymbol{f}(k) \geq 0$ hold.

Further, when (25) holds, we have

$$
\begin{aligned}
E\{\Delta V(\widetilde{\boldsymbol{x}}(k))\} & =E\{V(\widetilde{\boldsymbol{x}}(k+1)) \mid \tilde{\boldsymbol{x}}(k)\}-V(\widetilde{\boldsymbol{x}}(k)) \\
& \leq \zeta(k)^{T} \boldsymbol{\Phi} \zeta(k)<0
\end{aligned}
$$

Therefore, there exists a scalar $c$ such that the inequalities $E\left[V(\widetilde{\boldsymbol{x}}(k+1)-V(\widetilde{\boldsymbol{x}}(k))] \leq c\left\|\widetilde{\boldsymbol{x}}(k)^{2}\right\|\right.$ hold. According to the method proposed in [18], we can also prove that there exists a constant $\chi>0$ such that the inequalities $E\left[\sum_{k=0}^{\infty}\|\tilde{x}(k)\|^{2}\right]<$ $\chi \sup _{-\tau \leq i \leq 0} E\left[\|\phi(i)\|^{2}\right]$ hold. This means that system (18) is stochastically stable.

Secondly, when $\boldsymbol{w}(k) \neq 0$, we prove that system (18) is strictly $(\mathbf{Q}, \boldsymbol{S}, \boldsymbol{R})$ dissipative. 
Under zero initial state conditions, we define

$$
\begin{aligned}
E & \{\Delta V(\tilde{\boldsymbol{x}}(k))\}-E\left\{\boldsymbol{e}(k)^{T} \boldsymbol{Q} \boldsymbol{e}(k)+2 \boldsymbol{e}(k)^{T} \boldsymbol{S} \boldsymbol{w}(k)\right. \\
& \left.+\boldsymbol{w}(k)^{T}(\boldsymbol{R}-a \boldsymbol{I}) \boldsymbol{w}(k)\right\}+\tau \boldsymbol{x}(k)^{T} \boldsymbol{W}_{2}{ }^{T} \boldsymbol{W}_{2} \boldsymbol{x}(k) \\
& -\tau \boldsymbol{f}(k)^{T} \boldsymbol{f}(k)=\boldsymbol{\eta}(k)^{T}(\boldsymbol{\Lambda}+a \operatorname{diag}(0, \boldsymbol{I})) \boldsymbol{\eta}(k)
\end{aligned}
$$

where

$$
\boldsymbol{\eta}(k)=\left[\begin{array}{lll}
\tilde{\boldsymbol{x}}(k)^{T} & \boldsymbol{f}(k)^{T} & \boldsymbol{w}(k)^{T}
\end{array}\right]^{T},
$$

$\Lambda$

$$
\begin{aligned}
= & {\left[\begin{array}{ccc}
-\boldsymbol{P}+\boldsymbol{H} & * & * \\
\mathbf{0} & -\tau \boldsymbol{I} & * \\
-\boldsymbol{S}^{T} \widetilde{\boldsymbol{C}}^{*} & \mathbf{0} & -\boldsymbol{S}^{T} \boldsymbol{D}^{*}-\boldsymbol{D}^{* T} \boldsymbol{S}-\boldsymbol{R}
\end{array}\right] } \\
& -\left[\begin{array}{c}
\boldsymbol{G}_{1}^{T} \\
\boldsymbol{G}_{2}^{T} \\
\boldsymbol{G}_{3}^{T}
\end{array}\right] \widetilde{\boldsymbol{P}}^{-1}\left[\begin{array}{lll}
\boldsymbol{G}_{1} & \boldsymbol{G}_{2} & \boldsymbol{G}_{3}
\end{array}\right],
\end{aligned}
$$$$
\widetilde{\boldsymbol{P}}=\operatorname{diag}\left\{-\boldsymbol{P}^{-1},-\boldsymbol{P}^{-1},-\boldsymbol{P}^{-1},-\boldsymbol{P}^{-1}, \mathbf{Q}^{-1}, \mathbf{Q}^{-1}\right\},
$$$$
\boldsymbol{G}_{1}
$$$$
=\left[\begin{array}{llllll}
\boldsymbol{A}^{* T} & \sigma_{1} \widetilde{\boldsymbol{A}}_{1}^{T} & \sigma_{2} \widetilde{\boldsymbol{A}}_{2}^{T} & \sigma_{3}\left(\widetilde{\boldsymbol{A}}_{1}-\widetilde{\boldsymbol{A}}_{2}\right)^{T} & \boldsymbol{C}^{* T} & \sigma_{4} \widetilde{\boldsymbol{C}}_{1}^{T}
\end{array}\right]^{T}
$$

$\mathbf{G}_{3}$

$$
=\left[\begin{array}{lllll}
\boldsymbol{B}^{* T} & \sigma_{1} \widetilde{\boldsymbol{B}}_{1}^{T} & \sigma_{2} \widetilde{\boldsymbol{B}}_{1}^{T} & \sigma_{3}\left(\widetilde{\boldsymbol{B}}_{1}-\widetilde{\boldsymbol{B}}_{2}\right)^{T} \quad \boldsymbol{D}^{* T} & \sigma_{4} \widetilde{\boldsymbol{D}}^{T}
\end{array}\right]^{T},
$$$$
\boldsymbol{G}_{2}=\left[\begin{array}{lllll}
\widetilde{\boldsymbol{G}}^{T} & \mathbf{0} & \mathbf{0} & \mathbf{0} & \mathbf{0}
\end{array}\right]^{T} \text {. }
$$

According to Schur complement lemma, $\boldsymbol{\Lambda}<0$ is equivalent to (21).
When $\Lambda<0$, there must exist a sufficiently small scalar $a>0$ such that the inequalities $(\Lambda+a \operatorname{diag}(0, \boldsymbol{I}))<0$ hold. This means that (27) is less than zero.

Similar to the proof in the first part, we have

$$
\begin{gathered}
E\{\Delta V(\widetilde{\boldsymbol{x}}(k))\}-E\left\{\boldsymbol{e}(k)^{T} \boldsymbol{Q} \boldsymbol{e}(k)+2 \boldsymbol{e}(k)^{T} \boldsymbol{S} \boldsymbol{w}(k)\right. \\
\left.+\boldsymbol{w}(k)^{T}(\boldsymbol{R}-a \boldsymbol{I}) \boldsymbol{w}(k)\right\}<0
\end{gathered}
$$

Further, summing $k$ in (29) from 0 to $t$, we can obtain

$$
\begin{aligned}
E\{ & V(\tilde{\boldsymbol{x}}(T+1)\}-E\left\{V(\tilde{\boldsymbol{x}}(0)\}+E\left[a \sum_{0}^{t} \boldsymbol{w}(k)^{T} \boldsymbol{w}(k)\right]\right. \\
& <\bar{E}(w, e, t) .
\end{aligned}
$$

Considering that system (18) is stochastically stable and $V(\tilde{\boldsymbol{x}}(0))=0$ and $V(\tilde{\boldsymbol{x}}(T+1)) \geq 0$ under zero initial state conditions, we have $E\left[a \sum_{0}^{t} \boldsymbol{w}(k)^{T} \boldsymbol{w}(k)\right]<\bar{E}(w, e, t)$. This means that system (18) is strictly $(\boldsymbol{Q}, \boldsymbol{S}, \boldsymbol{R})$ dissipative.

This completes the proof.

\subsection{Solution of Filter Parameters}

Theorem 3. For given correlated perturbation parameter matrices $\widehat{\boldsymbol{H}}_{1} \in R^{(n+2 p) \times r}, \widehat{\boldsymbol{H}}_{2} \in R^{(n+2 p) \times r}, \boldsymbol{H}_{3} \in R^{n \times r}, \boldsymbol{H}_{4} \in$ $R^{q \times r}, \widehat{\boldsymbol{E}}_{1} \in R^{r \times(n+2 p)}, \boldsymbol{E}_{2} \in R^{r \times(n+2 p)}, \widehat{\boldsymbol{E}}_{3} \in R^{r \times(n+2 p)}, \boldsymbol{E}_{4} \in$ $R^{r \times p}$ and matrices $\mathbf{Q}=\mathbf{Q}^{T}<0, \boldsymbol{R}=\boldsymbol{R}^{T}$, and $\boldsymbol{S}$, if there exist constants $\varepsilon>0, \varepsilon_{i}>0(i=1,2,3,4,5)$ and $\tau>0$ and matrices $\boldsymbol{X} \in R^{(n+2 p) \times(n+2 p)}, \boldsymbol{Z} \in R^{(n+2 p) \times(n+2 p)}, \hat{\boldsymbol{A}}_{f} \in R^{(n+2 p) \times(n+2 p)}$, $\hat{\boldsymbol{B}}_{f} \in R^{(n+2 p) \times p}, \hat{\boldsymbol{C}}_{f} \in R^{q \times(n+2 p)}$, and $\hat{\boldsymbol{D}}_{f} \in R^{q \times p}$, the following LMI holds:

$$
\left[\begin{array}{ll}
\boldsymbol{\varphi}_{1} & \boldsymbol{\varphi}_{2}^{T} \\
\boldsymbol{\varphi}_{2} & \boldsymbol{\varphi}_{3}
\end{array}\right]<0
$$

Then system (18) is stochastically stable and strictly $(\mathbf{Q}, \mathbf{S}, \mathbf{R})$ dissipative, where

$$
\begin{aligned}
& \boldsymbol{\varphi}_{1}=\left[\begin{array}{ll}
\boldsymbol{\Xi}_{1} & \boldsymbol{\Xi}_{2}^{T} \\
\boldsymbol{\Xi}_{2} & \boldsymbol{\Xi}_{3}
\end{array}\right], \\
& \boldsymbol{\varphi}_{2}=\left[\begin{array}{ll}
\boldsymbol{O}_{1} & \boldsymbol{O}_{2} \\
\boldsymbol{O}_{3} & \boldsymbol{O}_{4}
\end{array}\right] \\
& \boldsymbol{\varphi}_{3}=\operatorname{diag}\left\{-\varepsilon_{1} \boldsymbol{I},-\varepsilon_{1} \boldsymbol{I},-\varepsilon_{1} \boldsymbol{I},-\varepsilon_{1} \boldsymbol{I},-\varepsilon_{2} \boldsymbol{I},-\varepsilon_{2} \boldsymbol{I},-\varepsilon_{3} \boldsymbol{I},-\varepsilon_{3} \boldsymbol{I},-\varepsilon_{4} \boldsymbol{I},-\varepsilon_{4} \boldsymbol{I},-\varepsilon_{5} \boldsymbol{I},-\varepsilon_{5} \boldsymbol{I}\right\} \\
& \boldsymbol{\Xi}_{1}=\left[\begin{array}{ll}
\boldsymbol{\Xi}_{11} & \boldsymbol{\Xi}_{12}^{T} \\
\boldsymbol{\Xi}_{12} & \boldsymbol{\Xi}_{13}
\end{array}\right], \\
& \boldsymbol{\Xi}_{11}=\left[\begin{array}{ccc}
-\boldsymbol{X}+\tau \boldsymbol{W}_{1}^{T} \boldsymbol{W}_{1}+\varepsilon \delta^{2} \boldsymbol{I}-\boldsymbol{Z}+\tau \boldsymbol{W}_{1}{ }^{T} \boldsymbol{W}_{1}+\varepsilon \delta^{2} \boldsymbol{I} & 0 \\
-\boldsymbol{Z}+\tau \boldsymbol{W}_{1}{ }^{T} \boldsymbol{W}_{1}+\varepsilon \delta^{2} \boldsymbol{I}-\boldsymbol{Z}+\tau \boldsymbol{W}_{1}{ }^{T} \boldsymbol{W}_{1}+\varepsilon \delta^{2} \boldsymbol{I} & 0 \\
0 & -\tau \boldsymbol{I}
\end{array}\right] \\
& \boldsymbol{\Xi}_{13}=\left[\begin{array}{cc}
\boldsymbol{M}_{1}{ }^{*} & \mathbf{0} \\
\boldsymbol{M}_{2}^{*} & \boldsymbol{M}_{3}{ }^{*}
\end{array}\right]
\end{aligned}
$$




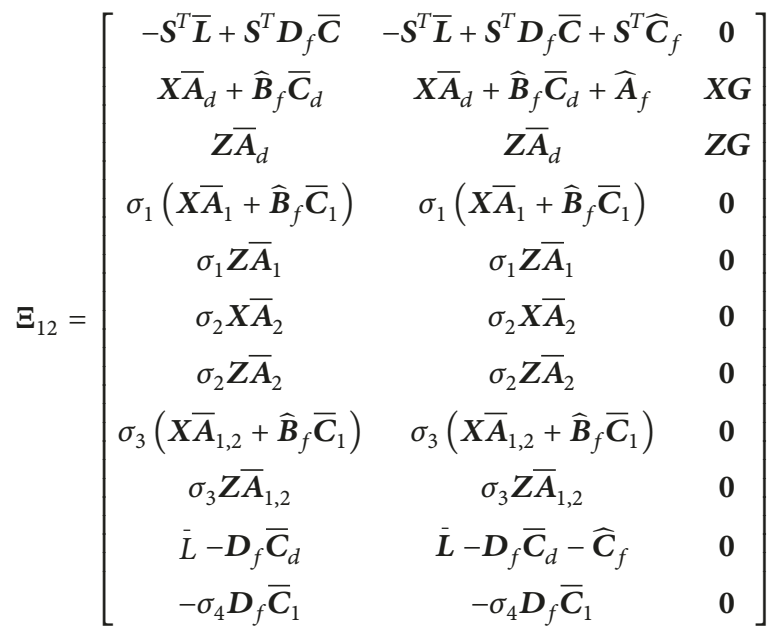

$\boldsymbol{M}_{1}^{*}=-\widetilde{\boldsymbol{D}}_{d}^{* T} \boldsymbol{S}-\boldsymbol{S}^{T} \widetilde{\boldsymbol{D}}_{d}^{* T}-\boldsymbol{R}+\varepsilon \delta^{2}$,

$\widetilde{\mathbf{D}}_{d}^{*}=\boldsymbol{L}_{2}-\overline{\mathbf{a}} \widehat{\mathbf{D}}_{f} \boldsymbol{D}$

$\boldsymbol{M}_{2}^{* T}$

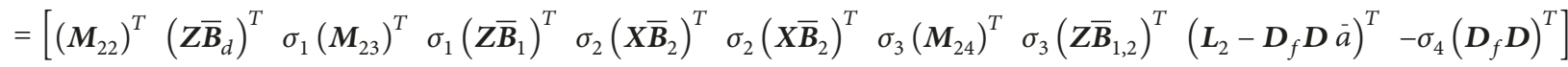

$\boldsymbol{M}_{22}=\boldsymbol{X} \overline{\boldsymbol{B}}_{d}+\widehat{\boldsymbol{B}}_{f} \boldsymbol{D} \bar{a}$,

$\boldsymbol{M}_{23}=\boldsymbol{X} \overline{\boldsymbol{B}}_{1}+\widehat{\boldsymbol{B}}_{f} \boldsymbol{D}$,

$\boldsymbol{M}_{24}=\boldsymbol{X} \overline{\boldsymbol{B}}_{1,2}+\widehat{\boldsymbol{B}}_{f} \boldsymbol{D}$

$\boldsymbol{M}_{3}{ }^{*}=\operatorname{diag}\left\{\boldsymbol{M}_{11}, \boldsymbol{M}_{11}, \boldsymbol{M}_{11}, \boldsymbol{M}_{11}, \boldsymbol{M}_{12}\right\}$

$M_{11}=\left[\begin{array}{ll}-\boldsymbol{X} & -\boldsymbol{Z} \\ -\boldsymbol{Z} & -\boldsymbol{Z}\end{array}\right]$,

$\boldsymbol{M}_{12}=\left[\begin{array}{cc}\mathbf{Q}^{-1} & \mathbf{0} \\ \mathbf{0} & \mathbf{Q}^{-1}\end{array}\right]$

$\Xi_{2}=\left[\begin{array}{ll}\Xi_{21} & \Xi_{22}\end{array}\right]$

$\Xi_{21}=\left[\begin{array}{cccccccc}\mathbf{0} & \mathbf{0} & \mathbf{0} & \boldsymbol{U}^{T} \boldsymbol{D}_{f d}^{T} \boldsymbol{S} & \bar{\alpha} \boldsymbol{M}_{1}^{T} \boldsymbol{X}+\bar{\alpha} \boldsymbol{M}_{11}^{T} \widehat{\boldsymbol{B}}_{f}^{T}+\bar{\delta} \boldsymbol{M}_{2}^{T} \boldsymbol{X} & \bar{\alpha} \boldsymbol{M}_{1}^{T} \boldsymbol{Z}+\bar{\delta} \boldsymbol{M}_{2}^{T} \boldsymbol{Z} & \sigma_{1} \boldsymbol{M}_{1}^{T} \boldsymbol{X}+\sigma_{1} \boldsymbol{M}_{11}^{T} \widehat{\boldsymbol{B}}_{f}^{T} & \sigma_{1} \boldsymbol{M}_{1}^{T} \boldsymbol{Z} \\ \mathbf{0} & \mathbf{0} & \mathbf{0} & \mathbf{0} & \mathbf{0} & \mathbf{0} & \mathbf{0} & \mathbf{0} \\ \mathbf{0} & \mathbf{0} & \mathbf{0} & \mathbf{0} & \mathbf{0} & \mathbf{0} & \mathbf{0} & \mathbf{0} \\ \mathbf{0} & \mathbf{0} & \mathbf{0} & \bar{\alpha} \boldsymbol{D}^{T} \boldsymbol{D}_{f d}^{T} \boldsymbol{S} & \bar{\alpha} \boldsymbol{N}_{1}^{T} \boldsymbol{X}+\bar{a} \boldsymbol{D}^{T} \widehat{\boldsymbol{B}}_{f}^{T}+\bar{\delta} \boldsymbol{N}_{2}^{T} \boldsymbol{X} & \bar{\alpha} \boldsymbol{N}_{1}^{T} \boldsymbol{Z} & \sigma_{1} \boldsymbol{N}_{1}^{T} \boldsymbol{X}+\sigma_{1} \boldsymbol{D}^{T} \widehat{\boldsymbol{B}}_{f}^{T} & \sigma_{1} \boldsymbol{N}_{1}^{T} \boldsymbol{Z}\end{array}\right]$

$\Xi_{22}=\left[\begin{array}{cccccc}\sigma_{2} \boldsymbol{M}_{2}^{T} \boldsymbol{X} & \sigma_{2} \boldsymbol{M}_{2}^{T} \boldsymbol{Z} & \sigma_{3} \boldsymbol{M}_{1}^{T} \boldsymbol{X}+\sigma_{3} \boldsymbol{M}_{11}^{T} \widehat{\boldsymbol{B}}_{f}^{T}-\sigma_{3} \boldsymbol{M}_{2}^{T} \boldsymbol{X} & \sigma_{3} \boldsymbol{M}_{1}^{T} \boldsymbol{Z}-\sigma_{3} \boldsymbol{M}_{2}^{T} \boldsymbol{Z} & -\bar{\alpha} \boldsymbol{U}^{T} \boldsymbol{D}_{f}^{T} & -\sigma_{4} \boldsymbol{U}^{T} \boldsymbol{D}_{f}^{T} \\ \mathbf{0} & \mathbf{0} & \mathbf{0} & \mathbf{0} & \mathbf{0} & \mathbf{0} \\ \mathbf{0} & \mathbf{0} & \mathbf{0} & \mathbf{0} & \mathbf{0} & \mathbf{0} \\ \sigma_{2} \boldsymbol{N}_{2}^{T} \boldsymbol{X} & \mathbf{0} & \sigma_{3} \boldsymbol{N}_{1}^{T} \boldsymbol{X}+\sigma_{3} \boldsymbol{D}^{T} \widehat{\boldsymbol{B}}_{f}^{T}-\sigma_{3} \boldsymbol{N}_{2}^{T} \boldsymbol{X} & \sigma_{3} \boldsymbol{N}_{1}^{T} \boldsymbol{Z} & -\bar{\alpha} \boldsymbol{D}^{T} \boldsymbol{D}_{f}^{T} & -\sigma_{4} \boldsymbol{D}^{T} \boldsymbol{D}_{f}^{T}\end{array}\right]$

$M_{1}=\left[\begin{array}{lll}0 & 0 & 0 \\ C & 0 & 0 \\ C & 0 & 0\end{array}\right]$,

$M_{2}=\left[\begin{array}{lll}0 & 0 & 0 \\ C & 0 & 0 \\ 0 & 0 & 0\end{array}\right]$, 


$$
\begin{aligned}
& N_{1}=\left[\begin{array}{l}
0 \\
D \\
D
\end{array}\right] \text {, } \\
& \boldsymbol{N}_{2}=\left[\begin{array}{l}
\mathbf{0} \\
\boldsymbol{D} \\
\mathbf{0}
\end{array}\right] \text {, } \\
& U=\left[\begin{array}{lll}
C & 0 & 0
\end{array}\right] \\
& \boldsymbol{\Xi}_{3}=\left[\begin{array}{cccc}
-\varepsilon \boldsymbol{I} & 0 & 0 & 0 \\
0 & -\varepsilon \boldsymbol{I} & 0 & 0 \\
0 & 0 & -\varepsilon \boldsymbol{I} & 0 \\
0 & 0 & 0 & -\varepsilon \boldsymbol{I}
\end{array}\right] \\
& \bar{A}_{1}=\left[\begin{array}{ccc}
0 & 0 & 0 \\
C & -I & 0 \\
C & -I & 0
\end{array}\right], \\
& \bar{B}_{1}=\left[\begin{array}{l}
0 \\
D \\
D
\end{array}\right] \\
& \bar{B}_{2}=\left[\begin{array}{l}
0 \\
D \\
0
\end{array}\right] \text {, } \\
& \overline{\boldsymbol{C}}_{1}=\left[\begin{array}{lll}
\boldsymbol{C} & -\boldsymbol{I} & 0
\end{array}\right], \\
& \bar{A}_{2}=\left[\begin{array}{ccc}
0 & 0 & 0 \\
C & -I & 0 \\
0 & 0 & 0
\end{array}\right] \text {, } \\
& \bar{A}_{1,2}=\bar{A}_{1}-\bar{A}_{2}, \\
& \overline{\boldsymbol{B}}_{1,2}=\overline{\boldsymbol{B}}_{1}-\overline{\boldsymbol{B}}_{2} \\
& \overline{\boldsymbol{A}}_{d}=\boldsymbol{A}_{0}+\bar{\alpha} \overline{\boldsymbol{A}}_{1}+\bar{\delta} \overline{\boldsymbol{A}}_{2}, \\
& \overline{\boldsymbol{B}}_{d}=\boldsymbol{B}_{0}+\bar{\alpha} \overline{\boldsymbol{B}}_{1}+\bar{\delta} \overline{\boldsymbol{B}}_{2}, \\
& \overline{\boldsymbol{C}}_{d}=\boldsymbol{C}_{0}+\bar{\alpha} \overline{\boldsymbol{C}}_{1}, \\
& \bar{\delta}=(1-\bar{\alpha}) \bar{\beta} \\
& \boldsymbol{O}_{1}=\left[\begin{array}{ccccccccc}
\mathbf{0} & \mathbf{0} & \mathbf{0} & \mathbf{0} & \widehat{\boldsymbol{H}}_{2}^{T} & \mathbf{0} & \mathbf{0} & \mathbf{0} & \mathbf{0} \\
\varepsilon_{1} \boldsymbol{E}_{2} \overline{\boldsymbol{C}}_{d} & \varepsilon_{1} \boldsymbol{E}_{2} \overline{\boldsymbol{C}}_{d} & \mathbf{0} & \varepsilon_{1} \cdot \bar{\alpha} \boldsymbol{E}_{2} \boldsymbol{D} & \mathbf{0} & \mathbf{0} & \mathbf{0} & \mathbf{0} & \mathbf{0} \\
\mathbf{0} & \mathbf{0} & \mathbf{0} & \mathbf{0} & \mathbf{0} & \mathbf{0} & \sigma_{1} \widehat{H}_{2}^{T} & \mathbf{0} & \mathbf{0} \\
\varepsilon_{1} \boldsymbol{E}_{2} \overline{\boldsymbol{C}}_{1} & \varepsilon_{1} \boldsymbol{E}_{2} \overline{\boldsymbol{C}}_{1} & \mathbf{0} & \varepsilon_{1} \boldsymbol{E}_{2} \boldsymbol{D} & \mathbf{0} & \mathbf{0} & \mathbf{0} & \mathbf{0} & \mathbf{0} \\
\mathbf{0} & \mathbf{0} & \mathbf{0} & \mathbf{0} & \widehat{\boldsymbol{H}}_{1}^{T} & \mathbf{0} & \mathbf{0} & \mathbf{0} & \mathbf{0} \\
\mathbf{0} & \varepsilon_{2} \widehat{\boldsymbol{E}}_{1} & \mathbf{0} & \mathbf{0} & \mathbf{0} & \mathbf{0} & \mathbf{0} & \mathbf{0} & \mathbf{0}
\end{array}\right],
\end{aligned}
$$




$$
\begin{aligned}
& \boldsymbol{O}_{2}=\left[\begin{array}{ccccccccc}
\mathbf{0} & \mathbf{0} & \mathbf{0} & \mathbf{0} & \mathbf{0} & \mathbf{0} & \mathbf{0} & \mathbf{0} & \mathbf{0} \\
\mathbf{0} & \mathbf{0} & \mathbf{0} & \mathbf{0} & \mathbf{0} & \varepsilon_{1} \cdot \bar{\alpha} \boldsymbol{E}_{2} \boldsymbol{M}_{11} & \mathbf{0} & \mathbf{0} & \varepsilon_{1} \cdot \bar{\alpha} \boldsymbol{E}_{2} \boldsymbol{D} \\
\mathbf{0} & \sigma_{3} \widehat{\boldsymbol{H}}_{2}^{T} & \mathbf{0} & \mathbf{0} & \mathbf{0} & \mathbf{0} & \mathbf{0} & \mathbf{0} & \mathbf{0} \\
\mathbf{0} & \mathbf{0} & \mathbf{0} & \mathbf{0} & \mathbf{0} & \varepsilon_{1} \boldsymbol{E}_{2} \boldsymbol{M}_{11} & \mathbf{0} & \mathbf{0} & \varepsilon_{1} \boldsymbol{E}_{2} \boldsymbol{D} \\
\mathbf{0} & \mathbf{0} & \mathbf{0} & \mathbf{0} & \mathbf{0} & \mathbf{0} & \mathbf{0} & \mathbf{0} & \mathbf{0} \\
\mathbf{0} & \mathbf{0} & \mathbf{0} & \mathbf{0} & \mathbf{0} & \mathbf{0} & \mathbf{0} & \mathbf{0} & \mathbf{0}
\end{array}\right] \\
& \boldsymbol{O}_{3}=\left[\begin{array}{ccccccccc}
\mathbf{0} & \mathbf{0} & \mathbf{0} & \widehat{\boldsymbol{H}}_{3}^{T} S & \mathbf{0} & \mathbf{0} & \mathbf{0} & \mathbf{0} & \mathbf{0} \\
\mathbf{0} & \varepsilon_{3} \widehat{\boldsymbol{E}}_{3} & \mathbf{0} & \mathbf{0} & \mathbf{0} & \mathbf{0} & \mathbf{0} & \mathbf{0} & \mathbf{0} \\
\mathbf{0} & \mathbf{0} & \mathbf{0} & \boldsymbol{H}_{4}^{T} \boldsymbol{S} & \mathbf{0} & \mathbf{0} & \mathbf{0} & \mathbf{0} & 0 \\
\varepsilon_{4} \boldsymbol{E}_{4} \overline{\boldsymbol{C}}_{d} & \varepsilon_{4} E_{4} \overline{\boldsymbol{C}}_{d} & \mathbf{0} & \varepsilon_{4} \boldsymbol{E}_{4} D \bar{a} & \mathbf{0} & \mathbf{0} & \mathbf{0} & \mathbf{0} & \mathbf{0} \\
\mathbf{0} & \mathbf{0} & \mathbf{0} & \mathbf{0} & \mathbf{0} & \mathbf{0} & \mathbf{0} & \mathbf{0} & 0 \\
\varepsilon_{5} \boldsymbol{E}_{4} \overline{\boldsymbol{C}}_{1} & \varepsilon_{5} \boldsymbol{E}_{4} \overline{\boldsymbol{C}}_{1} & \mathbf{0} & \varepsilon_{5} \boldsymbol{E}_{4} \boldsymbol{D} & \mathbf{0} & \mathbf{0} & \mathbf{0} & \mathbf{0} & 0
\end{array}\right], \\
& \boldsymbol{O}_{4}=\left[\begin{array}{ccccccccc}
\mathbf{0} & \mathbf{0} & \mathbf{0} & -\widehat{\boldsymbol{H}}_{3}^{T} & \mathbf{0} & \mathbf{0} & \mathbf{0} & \mathbf{0} & \mathbf{0} \\
\mathbf{0} & \mathbf{0} & \mathbf{0} & \mathbf{0} & \mathbf{0} & \mathbf{0} & \mathbf{0} & \mathbf{0} & \mathbf{0} \\
\mathbf{0} & \mathbf{0} & \mathbf{0} & -\boldsymbol{H}_{4}^{T} & \mathbf{0} & \mathbf{0} & \mathbf{0} & \mathbf{0} & \mathbf{0} \\
\mathbf{0} & \mathbf{0} & \mathbf{0} & \mathbf{0} & \mathbf{0} & \varepsilon_{4} \boldsymbol{E}_{4} \boldsymbol{U} & \mathbf{0} & \mathbf{0} & \varepsilon_{4} \boldsymbol{E}_{4} \boldsymbol{D} \\
\mathbf{0} & \mathbf{0} & \mathbf{0} & \mathbf{0} & -\sigma_{4} \boldsymbol{H}_{4}^{T} & \mathbf{0} & \mathbf{0} & \mathbf{0} & \mathbf{0} \\
\mathbf{0} & \mathbf{0} & \mathbf{0} & \mathbf{0} & \mathbf{0} & \varepsilon_{5} \boldsymbol{E}_{4} \boldsymbol{U} & \mathbf{0} & \mathbf{0} & \varepsilon_{5} \boldsymbol{E}_{4} \boldsymbol{D}
\end{array}\right]
\end{aligned}
$$

If (31) has a solution, then the parameters of the nonfragile dissipative filter can be calculated as follows:

$$
\begin{aligned}
\boldsymbol{A}_{f} & =\boldsymbol{W}^{-1} \hat{\boldsymbol{A}}_{f} \boldsymbol{Z}^{-1} \boldsymbol{V}^{-T}, \\
\boldsymbol{B}_{f} & =\boldsymbol{W}^{-1} \hat{\boldsymbol{B}}_{f} \\
\boldsymbol{C}_{f} & =\hat{\boldsymbol{C}}_{f} \boldsymbol{Z}^{-1} \boldsymbol{V}^{-T}, \\
\boldsymbol{D}_{f} & =\boldsymbol{D}_{f} \\
\boldsymbol{H}_{1} & =\boldsymbol{W}^{-1} \hat{\boldsymbol{H}}_{1} \\
\boldsymbol{E}_{1} & =\hat{\boldsymbol{E}}_{1} \boldsymbol{Z}^{-1} \boldsymbol{V}^{-T} \\
\boldsymbol{H}_{2} & =\boldsymbol{W}^{-1} \hat{\boldsymbol{H}}_{2} \\
\boldsymbol{E}_{3} & =\hat{\boldsymbol{E}}_{3} \boldsymbol{Z}^{-1} \boldsymbol{V}^{-T}
\end{aligned}
$$

where $\boldsymbol{W}$ and $\boldsymbol{V}$ are nonsingular constant matrices that meet $\boldsymbol{W} \boldsymbol{V}^{T}=\boldsymbol{I}-\boldsymbol{X} \boldsymbol{Z}^{-1}$.
Proof. Define

$$
\begin{aligned}
\boldsymbol{P} & =\left[\begin{array}{cc}
\boldsymbol{X} & \boldsymbol{W} \\
\boldsymbol{W}^{T} & -\boldsymbol{W}^{T} \boldsymbol{Z}^{-1} \boldsymbol{V}^{-T}
\end{array}\right], \\
\boldsymbol{\Omega}_{1} & =\left[\begin{array}{cc}
\boldsymbol{M}_{1} & \mathbf{0} \\
\boldsymbol{B}_{f d} \boldsymbol{M}_{11} & \mathbf{0}
\end{array}\right], \\
\boldsymbol{\Omega}_{2} & =\left[\begin{array}{cc}
\boldsymbol{M}_{2} & \mathbf{0} \\
\mathbf{0} & \mathbf{0}
\end{array}\right], \\
\boldsymbol{\Gamma}_{1} & =\left[\begin{array}{cc}
\boldsymbol{N}_{1} \\
\boldsymbol{B}_{f d} \boldsymbol{D}
\end{array}\right], \\
\boldsymbol{\Gamma}_{2} & =\left[\begin{array}{c}
\boldsymbol{N}_{2} \\
\mathbf{0}
\end{array}\right] \\
\boldsymbol{\Gamma}_{3} & =\left[\begin{array}{ll}
-\boldsymbol{D}_{f d} \boldsymbol{U} & \mathbf{0}
\end{array}\right], \\
\boldsymbol{\Gamma}_{4} & =-\boldsymbol{D}_{f d} \boldsymbol{D},
\end{aligned}
$$

and then (21) is equivalent to the following LMI: 


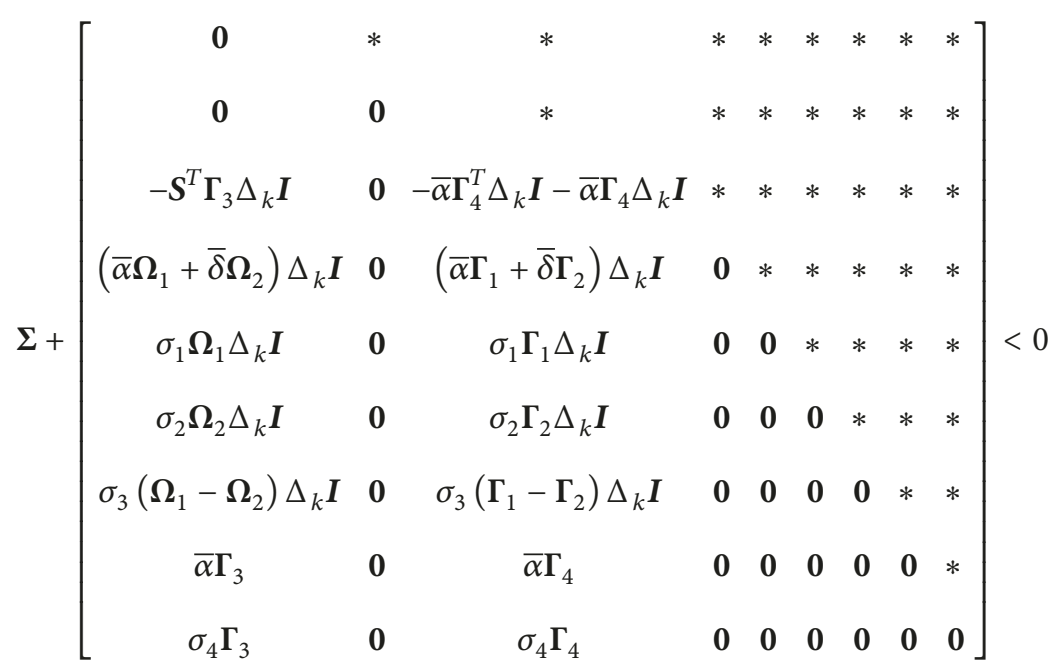

where

$$
\begin{aligned}
& \boldsymbol{\Sigma}=\left[\begin{array}{ll}
\boldsymbol{\Sigma}_{11} & \boldsymbol{\Sigma}_{12}^{T} \\
\boldsymbol{\Sigma}_{12} & \boldsymbol{\Sigma}_{22}
\end{array}\right], \\
& \Sigma_{11} \\
& =\left[\begin{array}{ccc}
-\boldsymbol{P}+\tau \boldsymbol{H}^{T} \boldsymbol{H}+\varepsilon \delta^{2} & * & * \\
\mathbf{0} & -\tau \boldsymbol{I} & * \\
-\boldsymbol{S}^{T} \widetilde{\boldsymbol{C}}_{d}^{*} & \mathbf{0} & -\widetilde{\boldsymbol{D}}_{d}^{* T} \boldsymbol{S}-\boldsymbol{S}^{T} \widetilde{\boldsymbol{D}}_{d}^{*}-\boldsymbol{R}+\varepsilon \delta^{2}
\end{array}\right] \\
& \boldsymbol{\Sigma}_{22}=\operatorname{diag}\left\{-\boldsymbol{P}^{-1},-\boldsymbol{P}^{-1},-\boldsymbol{P}^{-1},-\boldsymbol{P}^{-1}, \mathbf{Q}^{-1}, \mathbf{Q}^{-1}\right\} \\
& \Sigma_{12}^{T} \\
& =\left[\begin{array}{cccccc}
\widetilde{\boldsymbol{A}}_{d}^{* T} & \sigma_{1} \widetilde{\boldsymbol{A}}_{1 d}^{T} & \sigma_{2} \widetilde{\boldsymbol{A}}_{2 d}^{T} & \sigma_{3} \widetilde{\boldsymbol{A}}_{1,2 d}^{T} & \widetilde{\boldsymbol{C}}_{d}^{* T} & \sigma_{4} \widetilde{\boldsymbol{C}}_{1 d}{ }^{T} \\
\widetilde{\boldsymbol{G}}^{T} & \mathbf{0} & \mathbf{0} & \mathbf{0} & \mathbf{0} & \mathbf{0} \\
\widetilde{\boldsymbol{B}}_{d}^{* T} & \sigma_{1} \widetilde{\boldsymbol{B}}_{1 d}^{T} & \sigma_{2} \widetilde{\boldsymbol{B}}_{2 d}^{T} & \sigma_{3} \widetilde{\boldsymbol{B}}_{1,2 d}^{T} & \widetilde{\boldsymbol{D}}_{d}^{* T} & -\sigma_{4}\left(\boldsymbol{D}_{f} \boldsymbol{D}_{1 d}\right)^{T}
\end{array}\right] \\
& \widetilde{A}_{d}^{*}=\left[\begin{array}{cc}
\overline{A_{d}} & \mathbf{0} \\
\boldsymbol{B}_{f d} \bar{C}_{d} & A_{f d}
\end{array}\right] \text {, } \\
& \widetilde{\boldsymbol{B}}_{d}^{*}=\left[\begin{array}{cc}
\overline{\boldsymbol{B}}_{d} & \mathbf{0} \\
\boldsymbol{B}_{f d} & \overline{\boldsymbol{D}}_{d}
\end{array}\right], \\
& \widetilde{\boldsymbol{C}}_{d}^{*}=\left[\begin{array}{lll}
\overline{\mathbf{L}}-\boldsymbol{D}_{f d} & \overline{\boldsymbol{C}}_{d} & -\boldsymbol{C}_{f d}
\end{array}\right], \\
& \widetilde{\boldsymbol{A}}_{2 d}=\left[\begin{array}{cc}
\overline{\boldsymbol{A}}_{2} & \mathbf{0} \\
\mathbf{0} & \mathbf{0}
\end{array}\right] \text {, } \\
& \widetilde{\boldsymbol{A}}_{1 d}=\left[\begin{array}{cc}
\overline{\boldsymbol{A}}_{1} & \mathbf{0} \\
\boldsymbol{B}_{f d} \overline{\boldsymbol{C}}_{1} & \mathbf{0}
\end{array}\right] \text {, } \\
& \widetilde{\boldsymbol{B}}_{1 d}=\left[\begin{array}{c}
\overline{\boldsymbol{B}}_{1} \\
\boldsymbol{B}_{f d} \boldsymbol{D}
\end{array}\right] \text {, }
\end{aligned}
$$

$$
\begin{aligned}
& \widetilde{\boldsymbol{C}}_{1 d}=\left[\begin{array}{ll}
-\boldsymbol{D}_{f d} \overline{\boldsymbol{C}}_{1} & \mathbf{0}
\end{array}\right], \\
& \widetilde{\boldsymbol{A}}_{1,2 d}=\widetilde{\boldsymbol{A}}_{1 d}-\widetilde{\boldsymbol{A}}_{2 d} \\
& \widetilde{\boldsymbol{B}}_{1,2 d}=\widetilde{\boldsymbol{B}}_{1 d}-\widetilde{\boldsymbol{B}}_{2 d}, \\
& \widetilde{\boldsymbol{B}}_{2 d}=\left[\begin{array}{c}
\overline{\boldsymbol{B}}_{2} \\
\mathbf{0}
\end{array}\right] .
\end{aligned}
$$

Equation (35) can be simplified as $\boldsymbol{\Sigma}+\boldsymbol{H} \boldsymbol{M}+\boldsymbol{M}^{T} \boldsymbol{H}^{T}<0$, where

$$
\begin{aligned}
& \boldsymbol{H}=\left[\begin{array}{ccc}
\mathbf{0} & \mathbf{0} & \mathbf{0} \\
\mathbf{0} & \mathbf{0} & \mathbf{0} \\
-\boldsymbol{S}^{T} \boldsymbol{\Gamma}_{3} & \mathbf{0} & -\bar{a} \boldsymbol{\Gamma}_{4}^{T} \boldsymbol{I}-\bar{a} \boldsymbol{\Gamma}_{4} \\
\left(\bar{a} \boldsymbol{\Omega}_{1}+\bar{\delta} \boldsymbol{\Omega}_{2}\right) & \mathbf{0} & \left(\bar{a} \boldsymbol{\Gamma}_{1}+\bar{\delta} \boldsymbol{\Gamma}_{2}\right) \\
\sigma_{1} \boldsymbol{\Omega}_{1} & \mathbf{0} & \sigma_{1} \boldsymbol{\Gamma}_{1} \\
\sigma_{2} \boldsymbol{\Omega}_{2} & \mathbf{0} & \sigma_{2} \boldsymbol{\Gamma}_{2} \\
\sigma_{3}\left(\boldsymbol{\Omega}_{1}-\boldsymbol{\Omega}_{2}\right) & \mathbf{0} & \sigma_{3}\left(\boldsymbol{\Gamma}_{1}-\boldsymbol{\Gamma}_{2}\right) \\
\bar{a} \boldsymbol{\Gamma}_{3} & \mathbf{0} & \bar{a} \boldsymbol{\Gamma}_{4} \\
\sigma_{4} \boldsymbol{\Gamma}_{3} & \mathbf{0} & \sigma_{4} \boldsymbol{\Gamma}_{4}
\end{array}\right], \\
& \boldsymbol{M}=\left[\begin{array}{ccccccccc}
\Delta_{k} \boldsymbol{I} & 0 & 0 & 0 & 0 & 0 & 0 & 0 & 0 \\
0 & \Delta_{k} \boldsymbol{I} & 0 & 0 & 0 & 0 & 0 & 0 & 0 \\
0 & 0 & \Delta_{k} \boldsymbol{I} & 0 & 0 & 0 & 0 & 0 & 0
\end{array}\right]
\end{aligned}
$$

Considering that $\left\|\Delta_{k}\right\|^{2}<\delta^{2}$, based on Schur complement Lemma, we have

$$
\left[\begin{array}{ll}
\psi_{1} & \psi_{2}^{T} \\
\psi_{2} & \psi_{3}
\end{array}\right]<0
$$




$$
\begin{aligned}
& \psi_{1}=\left[\begin{array}{ll}
\prod_{1} & \prod_{3}^{T} \\
\prod_{3} & \prod_{2}^{2}
\end{array}\right] \\
& \prod_{1}=\left[\begin{array}{ccc}
-\boldsymbol{P}+\tau \boldsymbol{H}^{T} \boldsymbol{H}+\varepsilon \delta^{2} & * & * \\
\mathbf{0} & -\tau \boldsymbol{I} & * \\
-\boldsymbol{S}^{T} \widetilde{\boldsymbol{C}}_{d}^{*} & \mathbf{0} & -\widetilde{\boldsymbol{D}}_{d}^{* T} \boldsymbol{S}-\boldsymbol{S}^{T} \widetilde{\boldsymbol{D}}_{d}^{*}-\boldsymbol{R}+\varepsilon \delta^{2}
\end{array}\right] \text {, } \\
& \prod_{2}=\operatorname{diag}\left\{-\boldsymbol{P}^{-1},-\boldsymbol{P}^{-1},-\boldsymbol{P}^{-1},-\boldsymbol{P}^{-1}, \mathbf{Q}^{-1}, \mathbf{Q}^{-1}\right\} \text {, } \\
& \prod_{3}^{T}=\left[\begin{array}{cccccc}
\widetilde{\boldsymbol{A}}_{d}^{* T} & \sigma_{1} \widetilde{\boldsymbol{A}}_{1 d}^{T} & \sigma_{2} \widetilde{\boldsymbol{A}}_{2 d}^{T} & \sigma_{3} \widetilde{\boldsymbol{A}}_{1,2 d}^{T} & \widetilde{\boldsymbol{C}}_{d}^{* T} & \sigma_{4} \widetilde{\boldsymbol{C}}_{1 d}{ }^{T} \\
\widetilde{\boldsymbol{G}}^{T} & \mathbf{0} & \mathbf{0} & \mathbf{0} & \mathbf{0} & \mathbf{0} \\
\widetilde{\boldsymbol{B}}_{d}^{* T} & \sigma_{1} \widetilde{\boldsymbol{B}}_{1 d}^{T} & \sigma_{2} \widetilde{\boldsymbol{B}}_{2 d}^{T} & \sigma_{3} \widetilde{\boldsymbol{B}}_{1,2 d}^{T} & \widetilde{\boldsymbol{D}}_{d}^{* T} & -\sigma_{4}\left(\boldsymbol{D}_{f} \boldsymbol{D}_{1 d}\right)^{T}
\end{array}\right] \\
& \psi_{2}=\left[\begin{array}{ccccccccc}
\mathbf{0} & \mathbf{0} & -\boldsymbol{\Gamma}_{3}^{T} \boldsymbol{S} & \bar{\alpha} \boldsymbol{\Omega}_{1}^{T}+\bar{\delta} \boldsymbol{\Omega}_{2}^{T} & \sigma_{1} \boldsymbol{\Omega}_{1}^{T} & \sigma_{2} \boldsymbol{\Omega}_{2}^{T} & \sigma_{3}\left(\boldsymbol{\Omega}_{1}-\boldsymbol{\Omega}_{2}\right) & \bar{\alpha} \boldsymbol{\Gamma}_{3}^{T} & \sigma_{4} \boldsymbol{\Gamma}_{3}^{T} \\
\mathbf{0} & \mathbf{0} & \mathbf{0} & \mathbf{0} & \mathbf{0} & \mathbf{0} & \mathbf{0} & \mathbf{0} & \mathbf{0} \\
\mathbf{0} & \mathbf{0} & -\bar{\alpha} \boldsymbol{\Gamma}_{4}^{T} \boldsymbol{S} & \left(\bar{\alpha} \boldsymbol{\Gamma}_{1}^{T}+\bar{\delta} \boldsymbol{\Gamma}_{2}^{T}\right) & \sigma_{1} \boldsymbol{\Gamma}_{1}^{T} & \sigma_{2} \boldsymbol{\Gamma}_{2}^{T} & \sigma_{3}\left(\boldsymbol{\Gamma}_{1}-\boldsymbol{\Gamma}_{2}\right) & \bar{\alpha} \boldsymbol{\Gamma}_{4}^{T} & \sigma_{4} \boldsymbol{\Gamma}_{4}^{T}
\end{array}\right], \\
& \psi_{3}=\left[\begin{array}{ccc}
-\varepsilon \boldsymbol{I} & 0 & 0 \\
0 & -\varepsilon \boldsymbol{I} & 0 \\
0 & 0 & -\varepsilon \boldsymbol{I}
\end{array}\right]
\end{aligned}
$$

Let $\boldsymbol{\Sigma}_{1}=\left[\begin{array}{cc}\boldsymbol{X} & \boldsymbol{I} \\ \boldsymbol{V}^{T} & \mathbf{0}\end{array}\right]$ and $\boldsymbol{\Sigma}_{2}=\left[\begin{array}{ll}\boldsymbol{I} & \boldsymbol{Z}^{-1} \\ \mathbf{0} & \boldsymbol{V}^{T}\end{array}\right] ;$ (38) can be simplified as (31) with the following steps:

Step 1. Premultiply (38) with $\operatorname{diag}\left\{\boldsymbol{\Sigma}_{2}^{T}, \boldsymbol{I}, \boldsymbol{I}, \boldsymbol{\Sigma}_{1}^{T}, \boldsymbol{\Sigma}_{1}^{T}, \boldsymbol{\Sigma}_{1}^{T}, \boldsymbol{\Sigma}_{1}^{T}, \boldsymbol{I}\right.$, $\boldsymbol{I}, \boldsymbol{I}, \boldsymbol{I}\}$.

Step 2. Postmultiply $\operatorname{diag}\left\{\boldsymbol{\Sigma}_{2}, \boldsymbol{I}, \boldsymbol{I}, \boldsymbol{\Sigma}_{1}, \boldsymbol{\Sigma}_{1}, \boldsymbol{\Sigma}_{1}, \boldsymbol{\Sigma}_{1}, \boldsymbol{I}, \boldsymbol{I}, \boldsymbol{I}, \boldsymbol{I}\right\}$ on the basis of Step 1.

Step 3. Premultiply $\operatorname{diag}\{\boldsymbol{I}, \boldsymbol{Z}, \boldsymbol{I}, \boldsymbol{I}, \boldsymbol{I}, \boldsymbol{Z}, \boldsymbol{I}, \boldsymbol{Z}, \boldsymbol{I}, \boldsymbol{Z}, \boldsymbol{I}, \boldsymbol{Z}, \boldsymbol{I}$, $\boldsymbol{I}, \boldsymbol{I}, \boldsymbol{I}, \boldsymbol{I}, \boldsymbol{I}\}$ on the basis of Step 2.

Step 4. Postmultiply $\operatorname{diag}\{\boldsymbol{I}, \boldsymbol{Z}, \boldsymbol{I}, \boldsymbol{I}, \boldsymbol{I}, \boldsymbol{Z}, \boldsymbol{I}, \boldsymbol{Z}, \boldsymbol{I}, \boldsymbol{Z}, \boldsymbol{I}, \boldsymbol{Z}, \boldsymbol{I}$, $\boldsymbol{I}, \boldsymbol{I}, \boldsymbol{I}, \boldsymbol{I}, \boldsymbol{I}\}$ on the basis of Step 3 .

Step 5. Separate certainty with uncertainty in Step 4, and repeatedly utilize Lemma 1 and Schur complement Lemma.

This completes the proof.

Remark 1. The solution method of linear inequality (31) is general and will bring less conservatism. We can always get the solutions.

Remark 2. According to Theorem 3, the filter parameters of the system can be obtained by the feasible solution of linear inequality (31). Random delays and packet losses are assumed to occur simultaneously in the networked systems. Compared with the results of considering only random time delays or packet losses, the derivation process is more complicated due to the increase of random variables in this paper.

\section{Numerical Simulation and Discussion}

To verify the effectiveness of the proposed filter, we adopt the following system parameters given in [18]:

$$
\boldsymbol{A}=\left[\begin{array}{ccc}
-0.2 & 0.1 & 0.4 \\
0.7 & -0.1 & 0.1 \\
-0.3 & -0.2 & -0.1
\end{array}\right],
$$

$\boldsymbol{B}=\left[\begin{array}{c}-1 \\ -1.5 \\ 0.5\end{array}\right]$,

$D=0.9$,

$C=\left[\begin{array}{lll}1 & -2 & 1\end{array}\right]$

$\boldsymbol{L}_{1}=\left[\begin{array}{lll}-2 & 1 & -2\end{array}\right]$,

$\boldsymbol{L}_{2}=0$

$\stackrel{\boldsymbol{H}}{1}_{1}=\left[\begin{array}{lllll}1 & 0 & 0 & 0 & 0 \\ 0 & 1 & 0 & 0 & 0 \\ 0 & 0 & 1 & 0 & 0 \\ 0 & 0 & 0 & 1 & 0 \\ 0 & 0 & 0 & 0 & 1\end{array}\right]$, 


$$
\begin{aligned}
& \hat{\boldsymbol{E}}_{1}=0.01 *\left[\begin{array}{ccccc}
-1 & 0 & 0 & 0 & -1 \\
1 & 0 & 0 & 0 & 1 \\
-1 & 0 & 0 & 1 & 0 \\
1 & 0 & 0 & 0 & -1 \\
-1 & 0 & 1 & 0 & 0
\end{array}\right], \\
& \hat{\boldsymbol{H}}_{2}=\left[\begin{array}{l}
1 \\
1 \\
1 \\
1 \\
1
\end{array}\right] \\
& \boldsymbol{E}_{2}=0.02 \\
& \boldsymbol{H}_{4}=1, \\
& \boldsymbol{E}_{4}=0.02 \\
& \boldsymbol{H}_{3}=\left[\begin{array}{lllll}
1 & 0 & 0 & 2
\end{array}\right]
\end{aligned}
$$$$
\hat{\boldsymbol{E}}_{3}=0.02 *\left[\begin{array}{ccccc}
1 & 0 & 0 & 0 & 1 \\
-1 & 0 & 0 & 0 & -1 \\
1 & 0 & 0 & 1 & 0 \\
-1 & 1 & 0 & 0 & 0 \\
1 & 0 & 1 & 0 & 0
\end{array}\right]
$$$$
\boldsymbol{W}_{2}=\left[\begin{array}{ccc}
0.01 & 0 & 0 \\
0 & 0.01 & 0 \\
0 & 0 & 0.01
\end{array}\right] \text {, }
$$$$
\boldsymbol{f}(k, \boldsymbol{x}(k))=\left[\begin{array}{l}
0.01 \sin \left(\boldsymbol{x}(k)^{1}\right) \\
0.01 \sin \left(\boldsymbol{x}(k)^{2}\right) \\
0.01 \sin \left(\boldsymbol{x}(k)^{3}\right)
\end{array}\right]
$$$$
\boldsymbol{F}_{1}(k)=\boldsymbol{F}_{3}(k)
$$$$
=\left[\begin{array}{ccccc}
\sin (0.1 k) & 0 & 0 & 0 & 0 \\
0 & \sin (0.1 k) & 0 & 0 & 0 \\
0 & 0 & \sin (0.1 k) & 0 & 0 \\
0 & 0 & 0 & \sin (0.1 k) & 0 \\
0 & 0 & 0 & 0 & \sin (0.1 k)
\end{array}\right]
$$$$
\boldsymbol{F}_{2}(k)=\boldsymbol{F}_{4}(k)=\sin (0.1 k)
$$

Here, we choose $\bar{\alpha}=0.7, \bar{\beta}=0.8$, and $\rho=0.95$. Assume that disturbance $\boldsymbol{w}(k)$ is in the form of $\sin (k) * e^{-0.1 k}$. The parameters of dissipative filter are set as $\boldsymbol{Q}=-\boldsymbol{I}, \boldsymbol{S}=0.5 \boldsymbol{I}$, and $\boldsymbol{R}=12 \boldsymbol{I}$. According to Theorem 3, the parameters of traditional quantized dissipative filter without parameter perturbation and the parameters of the nonfragile quantized dissipative filter with parameter perturbation can be obtained by Matlab LMI toolbox. Then, we can obtain the performance metric $\gamma$ of corresponding dissipative filter based on the formula $\gamma=\sum(\|e(k)\|) / \sum(\|w(k)\|)$.

The parameters and performance metric $\gamma$ of the nonfragile quantized dissipative with parameter perturbation filter are listed as follows:
$\boldsymbol{A}_{f}$

$=\left[\begin{array}{ccccc}-1.3330 & -1.4733 & 0.5060 & 0.6555 & 0.0009 \\ 0.6161 & 0.3589 & -0.1316 & -0.3262 & -0.0005 \\ -1.0025 & -1.7769 & 0.3872 & -0.7236 & -0.0003 \\ 0.0001 & -0.1066 & 0.0783 & 0.1667 & 0.0000 \\ -0.0388 & -0.0666 & 0.0254 & 0.0237 & 0.0000\end{array}\right]$,

$\boldsymbol{B}_{f}=\left[\begin{array}{c}-0.5688 \\ 0.1140 \\ -0.5597 \\ -0.2520 \\ -131.1128\end{array}\right]$

$C_{f}=\left[\begin{array}{lllll}-0.1075 & -0.4379 & 0.2480 & 0.4629 & 0.0002\end{array}\right]$,

$D_{f}=-0.1801$,

$\gamma=0.9893$

The parameters of traditional quantized dissipative filter without parameter perturbation are listed as follows:

$$
\begin{aligned}
& \boldsymbol{A}_{f} \\
& =\left[\begin{array}{ccccc}
-1.0905 & -1.1645 & 0.4671 & 0.4963 & 0.0013 \\
0.4857 & 0.2565 & -0.1398 & -0.1720 & -0.0008 \\
-0.8796 & -1.3818 & 0.2421 & -0.3477 & -0.0025 \\
-0.0634 & -0.2373 & 0.1196 & 0.1112 & 0.0000 \\
-0.0385 & -0.0611 & 0.0231 & 0.0179 & -0.0000
\end{array}\right], \\
& \boldsymbol{B}_{f}=\left[\begin{array}{c}
-0.5539 \\
0.1482 \\
-0.4579 \\
-0.3401 \\
-52.8181
\end{array}\right] \\
& \boldsymbol{C}_{f}=\left[\begin{array}{lllll}
-0.0619 & -0.4009 & 0.2350 & 0.3271 & 0.0005
\end{array}\right], \\
& \boldsymbol{D}_{f}=-0.1726
\end{aligned}
$$

Note that there exists parameter perturbation in real networked systems; that is, $\Delta \boldsymbol{A}_{f}, \Delta \boldsymbol{B}_{f}, \Delta \boldsymbol{C}_{f}$, and $\Delta \boldsymbol{D}_{f}$ are unequal to zero. We can obtain the corresponding performance metric of traditional quantized dissipative filter with parameter perturbation in real networked systems; that is, $\gamma=1.0233$. It can be seen that the performance metric of the nonfragile quantized dissipative filter is less than its counterpart in the traditional quantized dissipative filter without parameter perturbation. This means that the nonfragile quantized dissipative filter is of better disturbance rejection capability than the traditional quantized dissipative filter.

The curves of $\boldsymbol{z}_{f}(k)$ estimated by nonfragile quantized dissipative filter and the signal to be estimated $\boldsymbol{z}(k)$ are 
TABLE 1: Performance metric $\gamma$ under different PTD and PPL.

\begin{tabular}{lcccccc}
\hline $\bar{\alpha}$ & $\bar{\beta}$ & PTD & PPL & $\gamma$ of TQDF & \multicolumn{1}{c}{$\gamma$ of NFQDF } \\
\hline 0.6 & 0.7 & 0.112 & 0.288 & 1.1511 & 1.1101 \\
0.7 & 0.8 & 0.072 & 0.228 & 1.0233 & $\mathbf{0 . 9 8 9 3}$ \\
0.8 & 0.9 & 0.036 & 0.164 & 1.0013 & $\mathbf{0 . 9 3 7 5}$ \\
\hline
\end{tabular}

Note that PTD, PPL, TQDF, and NFQDF are the abbreviations of probability of time delays, probability of the packet loss, traditional quantized dissipative filter, and nonfragile quantized dissipative filter, respectively.

TABLE 2: Performance metric $\gamma$ under different QD.

\begin{tabular}{lccc}
\hline QD & RQE & $\gamma$ of TQDF & $\gamma$ of NFQDF \\
\hline 0.75 & {$[-0.1428,0.1428]$} & 1.3401 & $\mathbf{1 . 2 6 3 9}$ \\
0.85 & {$[-0.0811,0.0811]$} & 1.1243 & $\mathbf{1 . 0 6 2 3}$ \\
0.95 & {$[-0.0256,0.0256]$} & 1.0233 & $\mathbf{0 . 9 8 9 3}$ \\
\hline
\end{tabular}

Note that QD, RQE, TQDF, and NFQDF are the abbreviations of quantization density, range of quantization error, traditional quantized dissipative filter, and nonfragile quantized dissipative filter, respectively.

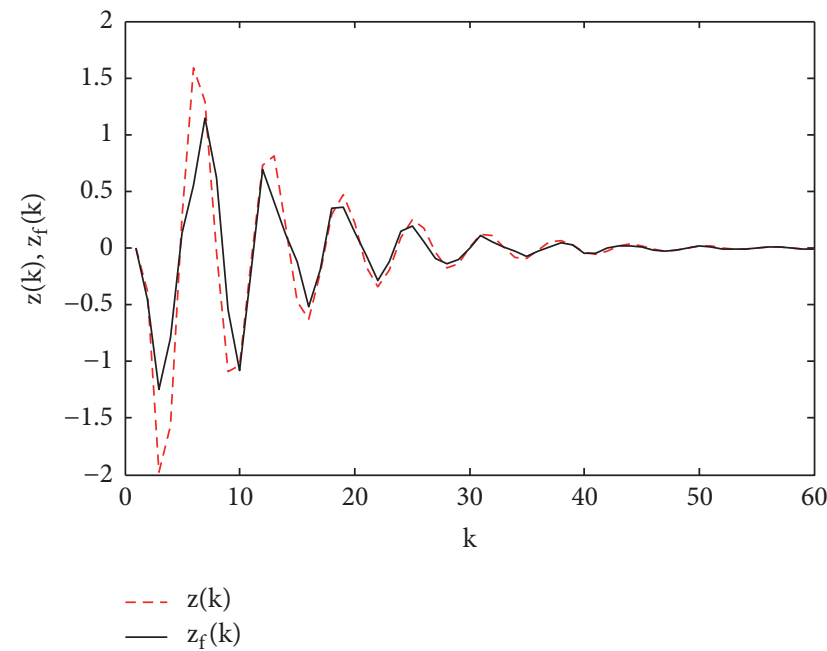

FIGURE 1: The curves of $\boldsymbol{z}_{f}(k)$ and $\boldsymbol{z}(k)$.

shown in Figure 1, respectively. We can see that $\boldsymbol{z}(k)$ will converge to $\boldsymbol{z}_{f}(k)$ within finite times $k$. This means that the designed nonfragile quantized dissipative filter is effective in real networked systems with time delays and packet losses.

We set $\rho=0.95$; considering the existence of different probability of time delays and packet losses in networked systems, the performance metric $\gamma$ of nonfragile quantized dissipative filter under the same quantization density is shown in Table 1. We can see that the nonfragile quantized dissipative filter is of better disturbance rejection capability than traditional quantized dissipative filter under given probability of time delays and packet losses. Furthermore, the performance metric $\gamma$ of nonfragile quantized dissipative filter will become larger and the estimation result of nonfragile quantized dissipative filter will become worse with the increase of the probability of time delays and packet losses. Therefore, the probability of time delays and packet losses may influence system performance.
We set $\bar{\alpha}=0.7, \bar{\beta}=0.8$; considering three different quantization densities, the performance metric $\gamma$ of nonfragile quantized dissipative filter under the same probability of time delays and packet losses is shown in Table 2. We can see that the nonfragile quantized dissipative filter is of better disturbance rejection capability than traditional quantized dissipative filter under given quantization density. Furthermore, the performance metric $\gamma$ of nonfragile quantized dissipative filter will become larger and the estimation result of nonfragile quantized dissipative filter will become worse with the decrease of quantization density. Therefore, the quantization density may also influence system performance.

\section{Conclusion}

In this paper, we investigate the problem of nonfragile quantized dissipative filter with parameter perturbation in nonlinear networked systems. Compared with the designed filter in [12-20], the designed nonfragile quantized dissipative filter in this paper considers the coexistence of time delays, packet losses, and quantization error in the real networked systems. The designed filter decreases the conservation problem by considering filter parameter perturbation. We obtain the sufficient conditions for the existence of nonfragile quantized dissipative filter by constructing appropriate Lyapunov function and utilizing linear matrix inequality method. Furthermore, we obtain the expression of the filter parameters. The simulation results show that the designed nonfragile quantized dissipative filter is better than traditional quantized dissipative filter without parameter perturbation.

\section{Data Availability}

The data used to support the findings of this study are available from the corresponding author upon request.

\section{Conflicts of Interest}

The authors declare that they have no conflicts of interest. 


\section{Acknowledgments}

The authors acknowledge financial support for this work from the scientific research project of Wuxi Institute of Technology of China in 2018 (no. ZK201810) and the National Natural Science Foundation of China (no. 61773182).

\section{References}

[1] H. C. Yan, Z. Z. Su, and H. B. Shi, "Quantized Ho filtering for discrete-time networked systems with mixed delays and missing measurements," vol. 56, pp. 2655-2660, IEEE, Hefei, China, 2011.

[2] H. C. Yan, Z. Z. Su, and H. B. Shi, "Ho filtering for discretetime networked systems with quantization and multiple packet dropouts," International Conference on Control, Automation, Robotics and Vision, vol. 56, no. 11, pp. 2655-2660, 2011.

[3] W. Yang, M. Liu, and P. Shi, "Ho filtering for nonlinear stochastic systems with sensor saturation, quantization and random packet losses," Signal Processing, vol. 92, no. 6, pp. 13871396, 2012.

[4] S. Zhou, L. Wang, and W. X. Zheng, "Ho filter design for nonlinear parameter-varying systems with quantized measurements," Journal of The Franklin Institute, vol. 349, no. 5, pp. 17811807, 2012.

[5] W.-W. Che, J.-L. Wang, and G.-H. Yang, "Quantised Ho filtering for networked systems with random sensor packet losses," Chinese Control and Descision Conference, vol. 4, no. 8, pp. 1339-1352, 2010.

[6] P. P. Wang and W. W. Che, "Quantized Ho filter design for networked control systems with random nonlinearity and sensor saturation," Neurocomputing, vol. 16, Article ID 825143, pp. 1-16, 2015.

[7] X.-M. Zhang and Q.-L. Han, "Network-based $H_{\infty}$ filtering using a logic jumping-like trigger," Automatica, vol. 49, no. 5, pp. 1428-1435, 2013.

[8] S.-P. Xiao, H.-H. Lian, K. L. Teo, H.-B. Zeng, and X.-H. Zhang, "A new Lyapunov functional approach to sampled-data synchronization control for delayed neural networks," Journal of The Franklin Institute, vol. 355, no. 17, pp. 8857-8873, 2018.

[9] S.-P. Xiao, H.-H. Lian, H.-B. Zeng, G. Chen, and W.-H. Zheng, "Analysis on robust passivity of uncertain neural networks with time-varying delays via free-matrix-based integral inequality," International Journal of Control, Automation, and Systems, vol. 15, no. 5, pp. 2385-2394, 2017.

[10] W. Wang, Q. B. Lin, and F. H. Cai Feng, "Robust passive filtering for a polytopic uncertain networked system," Systems Enineering and Electronics, vol. 5, no. 5, pp. 625-631, 2008.

[11] X. M. Zhang and Q. L. Han, "A Decentralized Event-Triggered Dissipative Control Scheme for Systems With Multiple Sensors to Sample the System Outputs," IEEE Transactions on Cybernetics, vol. 46, no. 12, pp. 27-45, 2016.

[12] Y. C. Ma, D. D. Cheng, and Y.-X. Zhao, "Robust Dissipative filter for a class of discrete time-delay nonlinear systems with parameter uncertainties," Control Engineering of China, vol. 19, no. 2, pp. 229-231, 2012.

[13] S. Zhou and G. Feng, "Ho filtering for discrete-time systems with randomly varying sensor delays," Automatica, vol. 44, no. 7, pp. 1918-1922, 2008.

[14] Q. B. Lin, F. W. Yang, and W. Wang, "Dissipative fuzzy filtering for nonlinear systems with multiple packet dropouts," Control Theory \& Applications, vol. 27, no. 3, pp. 363-368, 2010.
[15] J. Wang, X.-M. Zhang, and Q.-L. Han, "Event-triggered generalized dissipativity filtering for neural networks with timevarying delays," IEEE Transactions on Neural Networks and Learning Systems, vol. 27, no. 1, pp. 77-88, 2015.

[16] J. Tao, H. Su, R. Lu, and Z.-G. Wu, "Dissipativity-based filtering of nonlinear periodic Markovian jump systems: The discretetime case," Neurocomputing, vol. 171, pp. 807-814, 2016.

[17] P. Zhang, Y. M. Fu, and G. R. Duan, "Robust dissipative filter for linear uncertain time-delay systems," Journal of Harbin Institute of Technology. Harbin Gongye Daxue Xuebao, vol. 39, no. 3, pp. 345-348, 366, 2007.

[18] X. F. Yang, W. Yang, and J. C. Liu, "Non-fragile strictly dissipative filter for discrete-time nonlinear systems with sectorbounded nonlinearities," in Proceedings of the control and Decision Conference, pp. 4483-4488, CCDC, Xuzhou, China.

[19] W. Xia, Y. Li, Y. Chu, S. Xu, and Z. Zhang, "Dissipative filter design for uncertain Markovian jump systems with mixed delays and unknown transition rates," Signal Processing, vol. 141, pp. 176-186, 2017.

[20] K. Mathiyalagan, J. H. Park, and R. Sakthivel, "Robust reliable dissipative filtering for networked control systems with sensor failure," IET Signal Processing, vol. 8, no. 8, pp. 809-822, 2014. 


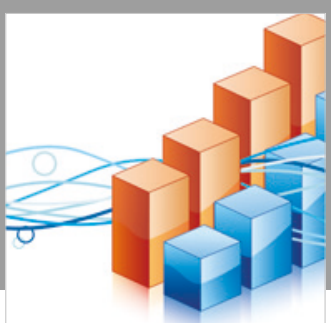

Advances in

Operations Research

\section{-n-m}
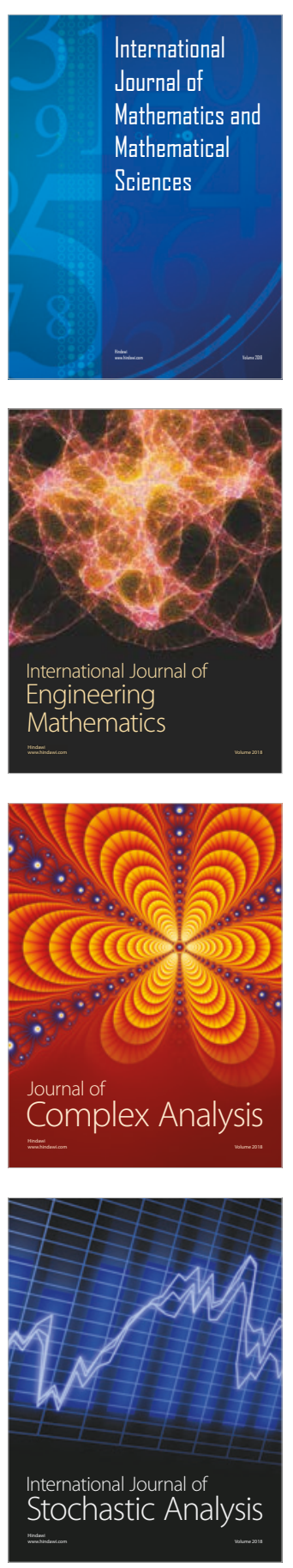
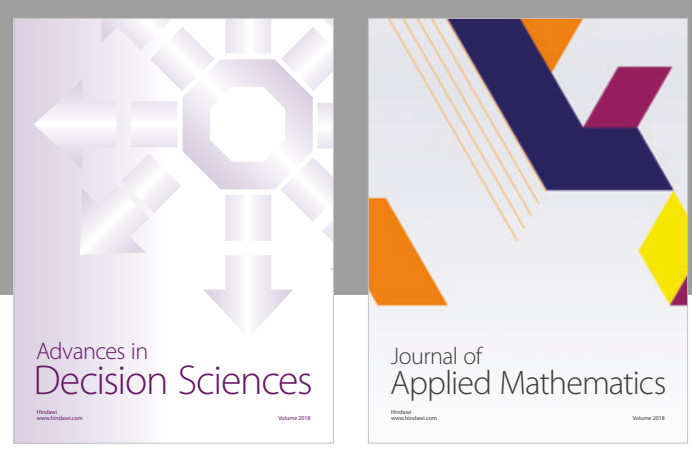

Journal of

Applied Mathematics
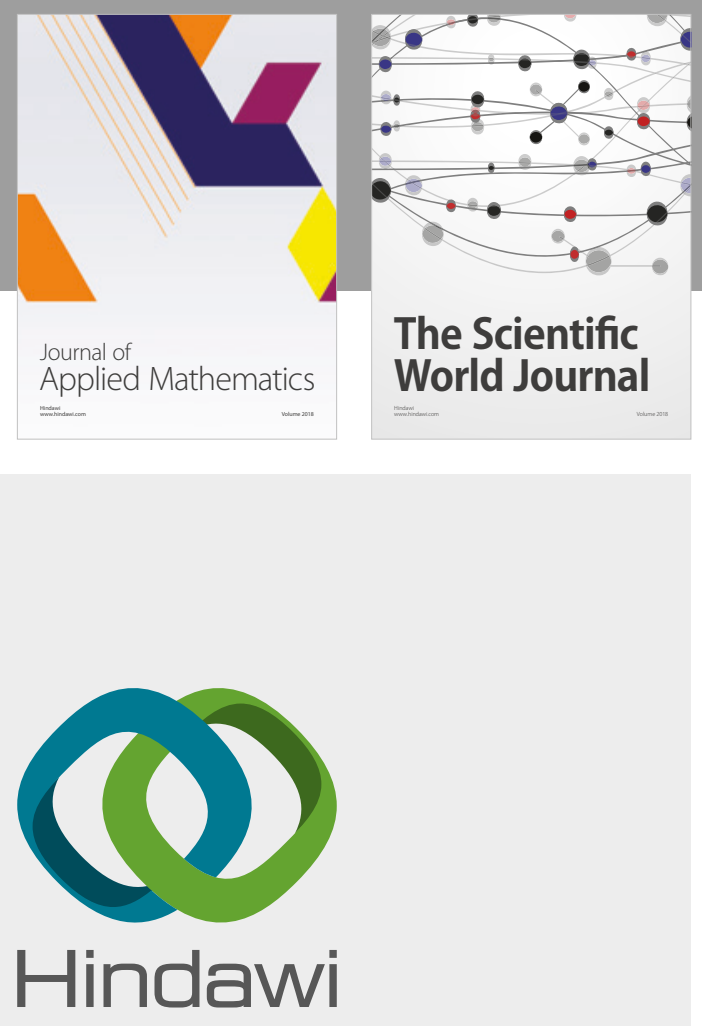

Submit your manuscripts at

www.hindawi.com

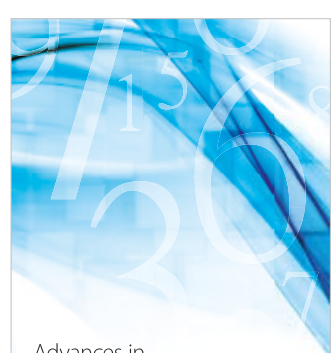

Advances in
Numerical Analysis
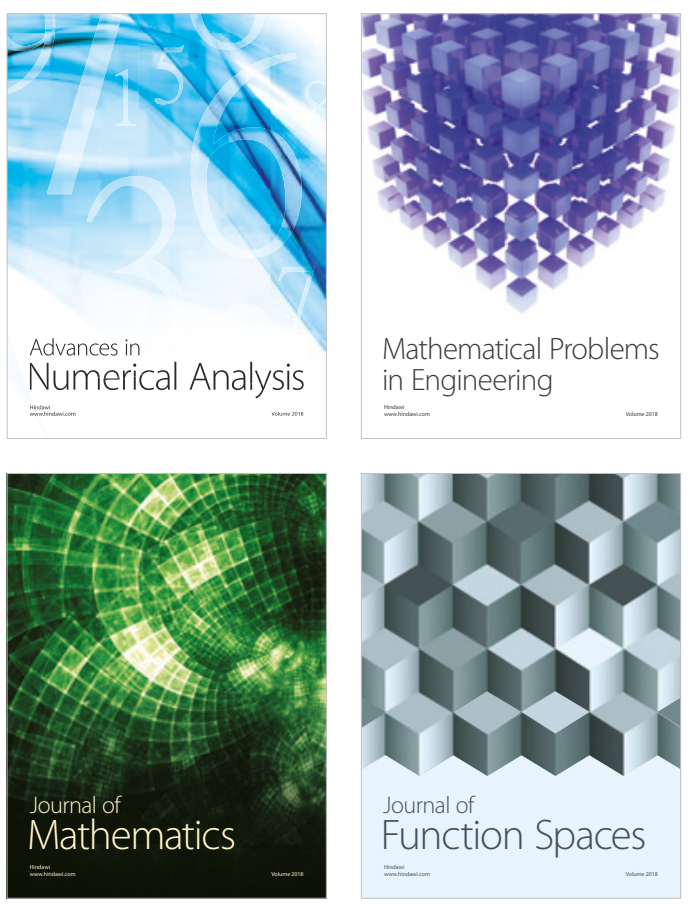

Mathematical Problems in Engineering

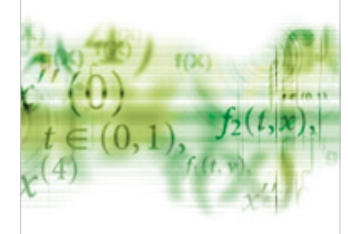

International Journal of

Differential Equations

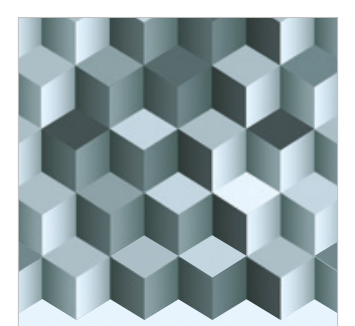

Journal of

Function Spaces

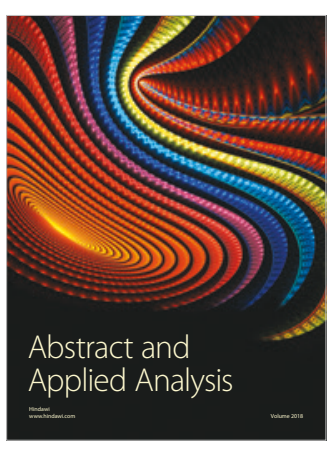

The Scientific

World Journal

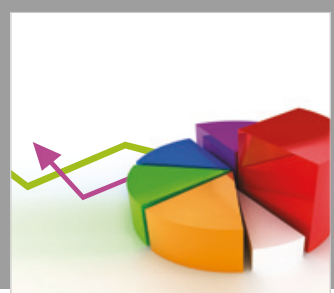

Journal of

Probability and Statistics
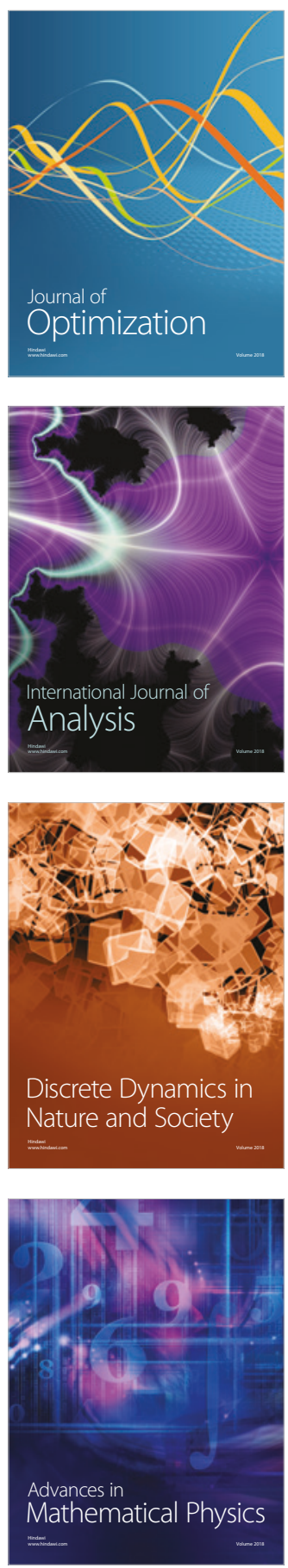